\title{
Size estimates for the EIT problem with one measurement: the complex case
}

\author{
Elena Beretta, Elisa Francini and Sergio Vessella
}

\begin{abstract}
In this paper we estimate the size of a measurable inclusion in terms of power measurements for a single applied boundary current. This problem arises in medical imaging for the screening of organs (see [17]). For this kind of problem one has to deal mathematically with the complex conductivity (admittivity) equation. In this case we are able to establish, for certain classes of admittivities, lower and upper bounds of the measure of the inclusion in terms of the power measurements. A novelty of our result is that we are also able to estimate the volume of an inclusion having part of its boundary in common with the reference body. Our analysis is based on the derivation of energy bounds and fine quantitative estimates of unique continuation for solutions to elliptic equations.
\end{abstract}

\section{Introduction}

In this paper we consider a mathematical problem arising in electrical impedance tomography (EIT), a nondestructive technique for determining electrical properties of a medium from measurements of voltages and currents at the boundary.

More precisely let $\Omega$ be the region occupied by a conducting medium and, at a fixed frequency $\omega$, consider the complex-valued admittivity function

$$
\gamma(x)=\sigma(x)+i \omega \epsilon(x)
$$

where $\sigma(x)$ represents the electrical conductivity at the point $x \in \Omega$ and $\epsilon(x)$ the electrical permittivity at a point $x \in \Omega$.

EIT leads to the inverse problem of the determination of the admittivity $\gamma$ from electrical measurements on $\partial \Omega$. This technique has several applications in medical imaging, nondestructive testing of materials and geophysical prospection of the underground. We refer to the review paper [9] and to [11] for an extensive bibliography comprising relevant examples of applications. For a variational approach of the admittivity equation see [12]. We point out that the admittivity equation also

Mathematics Subject Classification (2010): Primary 35R30; Secondary 35R25.

Keywords: Inverse boundary problems, size estimates, unique continuation. 
appears in the study of a model of electrical conduction in biological tissues as the asymptotic limit of an elliptic equation with memory subject to periodic Dirichlet boundary conditions (see [7] and [8]).

Relevant medical applications of EIT are for example breast cancer detection, (see for example [11]) and screening of organs in transplantation surgery ([17]). In these particular situations one can assume $\gamma$ to have the form

$$
\gamma=\gamma_{0} \chi_{\Omega \backslash D}+\gamma_{1} \chi_{D}
$$

where $D \subset \Omega$ is a measurable subset of $\Omega$ and $\gamma_{0} \neq \gamma_{1}$. Here $D$ represents the cancerous tissue or the degraded tissue which has a different admittivity than the surrounding healthy tissue represented by $\Omega \backslash D$. In particular in organ screening $D$ represents a region occupied by the degraded tissue imbedded in the healthy tissue and an important test to decide the quality of the organ is to give an estimate of the size of $D$ in terms of boundary observations ([17]).

We describe the mathematical problem: let $\Omega \subset \mathbb{R}^{n}, n \geq 2$, be a smooth, bounded domain and let $D \subset \Omega$ be a measurable subset of $\Omega$. We denote by $\gamma_{0}$ and $\gamma_{1}$ the admittivities of $\Omega \backslash D$ and $D$, respectively, with

$$
\gamma_{0}=\sigma_{0}+i \epsilon_{0} \quad \text { and } \quad \gamma_{1}=\sigma_{1}+i \epsilon_{1}
$$

(for simplicity we set $\omega=1$ ), we assume that

$$
\sigma_{0} \geq c_{0}>0, \quad \sigma_{1} \geq c_{0}>0
$$

this last condition corresponding to the dissipation of energy, and we let

$$
\gamma=\gamma_{0} \chi_{\Omega \backslash D}+\gamma_{1} \chi_{D}
$$

Let $h \in H^{-1 / 2}(\partial \Omega)$ be a complex-valued boundary current flux and consider the so-called background potential $u_{0} \in H^{1}(\Omega)$ generated by the flux $h$, which is the solution of

$$
\left\{\begin{aligned}
\operatorname{div}\left(\gamma_{0} \nabla u_{0}\right)=0 & & \text { in } \Omega \\
\gamma_{0} \frac{\partial u_{0}}{\partial \nu}=h & & \text { on } \partial \Omega
\end{aligned}\right.
$$

and let $u_{1} \in H^{1}(\Omega)$ be the perturbed potential generated by the flux $h$ in the presence of the inclusion $D$, which is the solution to

$$
\left\{\begin{aligned}
\operatorname{div}\left(\gamma \nabla u_{1}\right)=0 & \text { in } \Omega, \\
\gamma \frac{\partial u_{1}}{\partial \nu}=h & \text { on } \partial \Omega
\end{aligned}\right.
$$

where the systems defining $u_{0}$ and $u_{1}$ are subject to some combon normalization condition.

Consider now

$$
W_{1}=\int_{\partial \Omega} h \bar{u}_{1}
$$


which represents the power required to maintain the current $h$ in the presence of the inclusion $D$ and analogously define

$$
W_{0}=\int_{\partial \Omega} h \bar{u}_{0}
$$

the power required to maintain the current $h$ in the unperturbed medium. Let

$$
\delta W=W_{1}-W_{0}
$$

be the so called power gap.

We will show that, if the admittivities $\gamma_{0}$ and $\gamma_{1}$ are constant or if $\gamma_{0}$ and $\gamma_{1}$ are variable scalar admittivities with $\gamma_{0}$ satisfying $\Im \gamma_{0} \equiv 0$ and some extra conditions, then the measure $|D|$ of $D$ can be estimated in terms of $|\delta W|$. For, we follow the approach introduced in [5] and [6] where the authors derived estimates of $|D|$ in terms of the power gap for the real conductivity equation.

A different approach to deriving size estimates for real conductivity inclusions when $D$ comprises several connected components each of small size has been introduced in [10]. There the authors use multiple boundary measurements of a particular form to derive optimal asymptotic estimates of $D$. Recently Kang et al. (see [18]) obtained sharp bounds of the size of two dimensional conductivity inclusions from a pair of boundary measurements using classical variational principles.

We want to point out that in the the screening of organs it seems to be crucial to consider complex admittivities since electrical permittivity plays an important role in discriminating between degraded and normal tissue ([17]).

To derive our main results, as mentioned above, we follow the approach of [5] and [6] making use of the following basic tools:

- Energy bounds.

- Quantitative estimates of unique continuation and $A_{p}$ weights ([13]).

More precisely, the first step is to find energy bounds, i.e., lower and upper bounds for $\int_{D}\left|\nabla u_{0}\right|^{2}$ in terms of $|\delta W|$, and the second is to find lower and upper bounds for $\int_{D}\left|\nabla u_{0}\right|^{2}$ in terms of $|D|$ by using regularity and quantitative estimates of unique continuation of solutions to elliptic equations. Unfortunately, differently from the conductivity case, the first step in the complex case seems not to work for arbitrary admittivities but only for constant ones or for certain variable scalar admittivities (see assumption (H3) in Section 2).

On the other hand we would like to emphasize that, in [5] and [6], the authors make the following assumption

$$
d(D, \partial \Omega) \geq d_{0}>0
$$

Clearly this hypothesis is rather restrictive in the medical applications we have in mind since regions of the degraded tissue might extend to the surface of the organ. In this paper we remove this assumption and prove size estimates also for an inclusion having part of its boundary in common with $\partial \Omega$. This is accomplished by deriving fine quantitative estimates of unique continuation (Lemma 4.4), using reflection principles and suitable changes of variables. 
The paper is divided as follows. In Section 2 we state our main assumptions and our main results. In Section 3 we derive energy bounds of the form

$$
K_{1}|\delta W| \leq \int_{D}\left|\nabla u_{0}\right|^{2} \leq K_{2}|\delta W| .
$$

In Section 4 we list some useful tools concerning quantitative estimates of unique continuation. Section 5 is devoted to the proof of our main results. In particular we derive lower and upper bounds for the measure of the inclusion in terms of the energy of the background potential on $D$. Finally, in the appendix (Section 6), we give, for the reader's convenience, the proof of the doubling Inequality stated in Section 4.

\section{Main results}

\subsection{Notation and main assumptions}

For every $x \in \mathbb{R}^{n}$ we set $x=\left(x^{\prime}, x_{n}\right)$ where $x^{\prime} \in \mathbb{R}^{n-1}$ for $n \geq 2$.

Let $x_{0} \in \mathbb{R}^{n}$ and $r>0$. We denote by $B_{r}\left(x_{0}\right)$ and $B_{r}^{\prime}\left(x_{0}^{\prime}\right)$ the open ball in $\mathbb{R}^{n}$ centered at $x_{0}$ of radius $r$ and the open ball in $\mathbb{R}^{n-1}$ centered at $x^{\prime}$ of radius $r$, respectively. We denote by $Q_{l}\left(x_{0}\right)=\left\{x \in \mathbb{R}^{n}:\left|x_{j}-x_{0 j}\right| \leq l, j=1, \ldots, n\right\}$ the cube with center $x_{0}$ and side length $2 l$.

Definition 2.1 ( $C^{k, 1}$ regularity). Let $\Omega$ be a bounded domain in $\mathbb{R}^{n}$. Given $k$, with $k=0$, 1 , we say that $\partial \Omega$ or $\Omega$ is of class $C^{k, 1}$ with constants $r_{0}$ and $M_{0}$, if, for any $P \in \partial \Omega$, there exists a rigid transformation of coordinates under which $P=0$ and

$$
\Omega \cap\left\{B_{r_{0}}^{\prime}(0) \times\left(-M_{0} r_{0}, M_{0} r_{0}\right)\right\}=\left\{x \in B_{r_{0}}^{\prime}(0) \times\left(-M_{0} r_{0}, M_{0} r_{0}\right): x_{n}>\psi\left(x^{\prime}\right)\right\},
$$

where $\psi$ is a $C^{k, 1}$ function on $B_{r_{0}}^{\prime}(0)$ such that

$$
\psi(0)=0, \quad|\nabla \psi(0)|=0 \quad \text { when } k=1, \quad \text { and } \quad\|\psi\|_{C^{k, 1}\left(B_{r_{0}}^{\prime}\right)} \leq M_{0} r_{0} .
$$

For $z, w \in \mathbb{C}^{n}$ we write by $z \cdot w=\sum_{j=1}^{n} z_{j} w_{j}$.

Remark 2.2. Our convention is to normalize all norms so that that their terms are dimensionally homogeneous with respect to their argument and they coincide with the standard definitions when the dimension parameter equals one. For instance, the norm appearing above is meant as follows when $k=1$ :

$$
\|\psi\|_{C^{1,1}\left(B_{r_{0}}^{\prime}\right)}=\|\psi\|_{L^{\infty}\left(B_{r_{0}}^{\prime}\right)}+r_{0}\|\nabla \psi\|_{L^{\infty}\left(B_{r_{0}}^{\prime}\right)}+r_{0}^{2}|\nabla \psi|_{1, B_{r_{0}}^{\prime}},
$$

where

$$
|\nabla \psi|_{1, B_{r_{0}}^{\prime}}=\sup _{\substack{x, y \in B_{r_{0}}^{\prime} \\ x \neq y}} \frac{|\nabla \psi(x)-\nabla \psi(y)|}{|x-y|} .
$$


Similarly, given a function $u: \Omega \mapsto \mathbb{C}$,

$$
\|u\|_{L^{2}(\Omega)}=r_{0}^{-1}\left(\int_{\Omega}|u|^{2}\right)^{1 / 2}, \quad\|u\|_{H^{1}(\Omega)}=r_{0}^{-1}\left(\int_{\Omega}|u|^{2}+r_{0}^{2} \int_{\Omega}|\nabla u|^{2}\right)^{1 / 2},
$$

and so on for boundary and trace norms such as $\|\cdot\|_{H^{1 / 2}(\partial \Omega)}$ or $\|\cdot\|_{H^{-1 / 2}(\partial \Omega)}$.

We denote by $\Omega_{r}, r>0$, the set

$$
\Omega_{r}=\{x \in \Omega: \operatorname{dist}(x, \partial \Omega)>r\} .
$$

Let now state our main assumptions.

(H1) Assumptions on $\Omega$.

Let $M_{0}, M_{1}$ and $r_{0}$ be positive numbers such that $M_{0} \geq 1$. We assume that

1. $\Omega$ is a bounded domain in $\mathbb{R}^{n}$ with connected boundary;

2. $\partial \Omega$ has $C^{0,1}$ regularity with constants $r_{0}$ and $M_{0}$;

3. $|\Omega| \leq M_{1} r_{0}^{n}$.

(H2) Assumptions on D.

$D$ is a Lebesgue measurable subset of $\bar{\Omega}$ and

(H2a) there exists a positive constant $d_{0} \operatorname{such}$ that $\operatorname{dist}(D, \partial \Omega) \geq d_{0}$, or

(H2b) there exist $r_{1} \in\left(0, r_{0}\right]$ and $P \in \partial \Omega$ such that $D \subset \bar{\Omega} \backslash B_{r_{1}}(P)$.

(H3) Assumptions on the coefficients.

Let $c_{0} \in(0,1], \mu_{0}$, and $L$ be positive numbers. We assume the reference medium and the inclusion have admittivities $\gamma_{0}=\sigma_{0}+i \epsilon_{0}$ and $\gamma_{1}=\sigma_{1}+i \epsilon_{1}$ satisfying

$$
\sigma_{j} \geq c_{0},\left|\gamma_{j}\right| \leq c_{0}^{-1} \text { in } \Omega, \text { for } j=0,1,
$$

and, moreover we assume that:

(H3i) $\gamma_{0}$ and $\gamma_{1}$ are constants, and we set $\mu_{0}=\left|\gamma_{0}-\gamma_{1}\right|>0$, or

(H3ii) $\epsilon_{0}(x) \equiv 0$ in $\Omega$, and $\left|\sigma_{0}(x)-\sigma_{0}(y)\right| \leq \frac{L}{r_{0}}|x-y|$ for $x, y \in \Omega$, and

$$
\left|\epsilon_{1}(x)\right| \geq \mu_{0} \text { or } \sigma_{1}(x)-\sigma_{0}(x) \geq \mu_{0} \text { in } \Omega \text {. }
$$

(H4) Assumptions on the boundary data.

(H4a) Let $h \in H^{-1 / 2}(\partial \Omega)$ be a complex-valued nontrivial current density on $\partial \Omega$ satisfying

$$
\int_{\partial \Omega} h=0
$$


or

(H4b) Let $h \in H^{-1 / 2}(\partial \Omega)$ be a complex-valued nontrivial current density on $\partial \Omega$ satisfying

$$
\int_{\partial \Omega} h=0
$$

and such that

$$
\operatorname{supp} h \subset \Gamma_{0}:=\partial \Omega \cap \bar{B}_{r_{1} / 2}(P),
$$

for the same $r_{1}$ and $P$ as in assumption (H2b).

We denote by $F(h)$ the frequency of $h$, that is

$$
F(h)=\frac{\|h\|_{H^{-1 / 2}(\partial \Omega)}}{\|h\|_{H^{-1}(\partial \Omega)}} .
$$

Let

$$
\gamma=\gamma_{0} \chi_{\Omega \backslash D}+\gamma_{1} \chi_{D}
$$

and consider the unique solution $u_{1} \in H^{1}(\Omega)$ of the problem

$$
\left\{\begin{aligned}
\operatorname{div}\left(\gamma \nabla u_{1}\right) & =0 \quad \\
\gamma \frac{\partial u_{1}}{\partial \nu} & =h \quad \text { in } \Omega \\
\int_{\partial \Omega} u_{1} & =0 .
\end{aligned}\right.
$$

Analogously we define the background potential $u_{0} \in H^{1}(\Omega)$ generated by the same current flux $h$, to be the unique solution to the problem

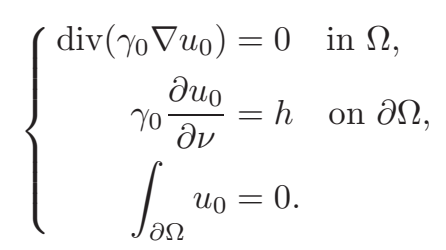

We shall denote by $W_{1}$ and $W_{0}$ the power necessary to maintain the current $h$ when $D$ is present or absent, respectively, so that

$$
W_{1}=\int_{\partial \Omega} h \bar{u}_{1}=\int_{\Omega} \gamma \nabla u_{1} \nabla \bar{u}_{1},
$$

and

$$
W_{0}=\int_{\partial \Omega} h \bar{u}_{0}=\int_{\Omega} \gamma_{0} \nabla u_{0} \nabla \bar{u}_{0} .
$$

Let $\delta W=W_{1}-W_{0}$ be the power gap. 


\subsection{The main theorems}

We first state our main result in the case of an inclusion $D$ strictly contained in $\Omega$.

Theorem 2.3. Let $\Omega$ satisfy $(\mathbf{H 1})$ and let $D$ be a measurable subset of $\Omega$ satisfying (H2a). Let $\gamma_{0}$ and $\gamma_{1}$ satisfy (H3) and let $h$ satisfy (H4a). Then,

$$
C_{1}\left|\frac{\delta W}{W_{0}}\right| \leq \frac{|D|}{|\Omega|} \leq C_{2}\left|\frac{\delta W}{W_{0}}\right|^{1 / p}
$$

where $C_{1}$ depends on the parameters $c_{0}, \mu_{0}, M_{0}, M_{1}, d_{0} / r_{0}$ and $L$, and the numbers $p>1$ and $C_{2}$ depend on the same parameters and, in addition, on $F(h)$.

We now state our main result in the case of of an inclusion that might have part of its boundary in common with $\partial \Omega$.

Theorem 2.4. Let $\Omega$ satisfy (H1) with $\partial \Omega \in C^{1,1}$ with constants $r_{0}$ and $M_{0}$ and let $D$ be a measurable subset of $\Omega$ satisfying $(\mathbf{H 2 b})$. Let $\gamma_{0}$ and $\gamma_{1}$ satisfy (H3) and let $h$ satisfy $(\mathbf{H} 4 \mathbf{b})$. Then,

$$
C_{1}\left|\frac{\delta W}{W_{0}}\right| \leq \frac{|D|}{|\Omega|} \leq C_{2}\left|\frac{\delta W}{W_{0}}\right|^{1 / p}
$$

where $C_{1}$ depends on the parameters $c_{0}, \mu_{0}, M_{0}, M_{1}, r_{1} / r_{0}$ and $L$, and the numbers $p>1$ and $C_{2}$ depend on the same parameters and, in addition, on $F(h)$.

The proofs of Theorem 2.3 and 2.4 will be given in Section 5 .

\section{Energy bounds}

\subsection{Energy identities}

In this section, following an idea first introduced in [19], we use energy identities in order to derive suitable energy bounds.

Let $\tilde{\gamma}$ be a complex admittivity and define the sesquilinear form

$$
a_{\tilde{\gamma}}(u, v)=\int_{\Omega} \tilde{\gamma} \nabla u \cdot \nabla \bar{v}
$$

If $u_{\tilde{\gamma}}$ is a solution to



then

$$
a_{\tilde{\gamma}}\left(u_{\tilde{\gamma}}, v\right)=\int_{\partial \Omega} h \bar{v}, \quad \forall v \in H^{1}(\Omega) .
$$

We observe that in general $a_{\tilde{\gamma}}$ is not complex symmetric:

$$
a_{\tilde{\gamma}}(u, v)-a_{\tilde{\gamma}}(v, u)=\int_{\Omega} \tilde{\gamma}(\nabla u \cdot \nabla \bar{v}-\nabla v \cdot \nabla \bar{u})=2 i \int_{\Omega} \tilde{\gamma} \Im(\nabla u \cdot \nabla \bar{v}) .
$$


Lemma 3.1. Let $\gamma_{0}$ and $\gamma_{1}$ be in $L^{\infty}(\Omega)$, let $\gamma=\gamma_{0} \chi_{\Omega \backslash D}+\gamma_{1} \chi_{D}$, and let $u_{1}$ and $u_{0}$ be the solutions of (2.2) and (2.3), respectively. The following identities hold:

$($ id1 $) \quad \int_{\Omega} \gamma\left|\nabla\left(u_{1}-u_{0}\right)\right|^{2}-\int_{D}\left(\gamma_{1}-\gamma_{0}\right)\left|\nabla u_{0}\right|^{2}=\delta W+2 i \int_{\Omega} \gamma \Im\left(\nabla u_{1} \cdot \nabla \bar{u}_{0}\right)$,

(id2) $\int_{\Omega} \gamma_{0}\left|\nabla\left(u_{1}-u_{0}\right)\right|^{2}+\int_{D}\left(\gamma_{1}-\gamma_{0}\right)\left|\nabla u_{1}\right|^{2}=-\delta W-2 i \int_{\Omega} \gamma_{0} \Im\left(\nabla u_{1} \cdot \nabla \bar{u}_{0}\right)$,

(id3)

$$
\begin{aligned}
& \int_{D}\left(\gamma_{0}-\gamma_{1}\right) \nabla u_{1} \cdot \nabla \bar{u}_{0}=\delta W+2 i \int_{\Omega} \gamma_{0} \Im\left(\nabla u_{1} \cdot \nabla \bar{u}_{0}\right) \\
& \int_{D}\left(\gamma_{1}-\gamma_{0}\right) \nabla u_{0} \cdot \nabla \bar{u}_{1}=-\delta W-2 i \int_{\Omega} \gamma \Im\left(\nabla u_{1} \cdot \nabla \bar{u}_{0}\right) .
\end{aligned}
$$

Proof. We write $a_{0}(u, v):=a_{\gamma_{0}}(u, v)$ and $a_{1}(u, v):=a_{\gamma}(u, v)$ From (3.1) we have

$$
a_{0}\left(u_{0}, v\right)=a_{1}\left(u_{1}, v\right)=\int_{\partial \Omega} h \bar{v}, \quad \forall v \in H^{1}(\Omega) .
$$

We compute

$$
\begin{aligned}
J_{1} & :=a_{1}\left(u_{1}-u_{0}, u_{1}-u_{0}\right)-\left[a_{1}\left(u_{0}, u_{0}\right)-a_{0}\left(u_{0}, u_{0}\right)\right] \\
& =\int_{\partial \Omega} h \bar{u}_{1}-\int_{\partial \Omega} h \bar{u}_{0}+2 i \int_{\Omega} \gamma \Im\left(\nabla u_{1} \cdot \nabla \bar{u}_{0}\right) .
\end{aligned}
$$

On the other hand,

$$
\begin{aligned}
J_{1} & =\int_{\Omega} \gamma\left|\nabla\left(u_{1}-u_{0}\right)\right|^{2}-\int_{\Omega}\left(\gamma-\gamma_{0}\right)\left|\nabla u_{0}\right|^{2} \\
& =\int_{\Omega} \gamma\left|\nabla\left(u_{1}-u_{0}\right)\right|^{2}-\int_{D}\left(\gamma_{1}-\gamma_{0}\right)\left|\nabla u_{0}\right|^{2}
\end{aligned}
$$

and so, by (3.2) and (3.3) and the definition of $\delta W$, the identity (id1) follows.

Analogously we can compute

$$
\begin{aligned}
J_{2} & :=a_{0}\left(u_{0}-u_{1}, u_{0}-u_{1}\right)-\left[a_{0}\left(u_{1}, u_{1}\right)-a_{1}\left(u_{1}, u_{1}\right)\right] \\
& =-\int_{\partial \Omega} h\left(\bar{u}_{1}-\bar{u}_{0}\right)-2 i \int_{\Omega} \gamma_{0} \Im\left(\nabla u_{1} \cdot \nabla \bar{u}_{0}\right) .
\end{aligned}
$$

On the other hand,

$$
J_{2}=\int_{\Omega} \gamma_{0}\left|\nabla\left(u_{1}-u_{0}\right)\right|^{2}+\int_{\Omega}\left(\gamma_{1}-\gamma_{0}\right)\left|\nabla u_{1}\right|^{2}
$$

and, hence, (id2) follows.

Finally let us compute

$$
a_{0}\left(u_{1}, u_{0}\right)-a_{1}\left(u_{1}, u_{0}\right)=\int_{D}\left(\gamma_{0}-\gamma_{1}\right) \nabla u_{1} \cdot \nabla \bar{u}_{0},
$$


and, observe that

$$
\begin{aligned}
a_{0}\left(u_{1}, u_{0}\right)-a_{1}\left(u_{1}, u_{0}\right) & =a_{0}\left(u_{1}, u_{0}\right)-a_{1}\left(u_{1}, u_{0}\right)+a_{0}\left(u_{0}, u_{1}\right)-a_{0}\left(u_{0}, u_{1}\right) \\
& =\int_{\partial \Omega} h\left(\bar{u}_{1}-\bar{u}_{0}\right)+2 i \int_{\Omega} \gamma_{0} \Im\left(\nabla u_{1} \cdot \nabla \bar{u}_{0}\right),
\end{aligned}
$$

so that (id3) follows.

By symmetry we have also shown (id4)

Remark 3.2. Note that by combining (id1) and (id4), we get as an easy consequence of the definition of $u_{0}$ and $u_{1}$, that

$$
\int_{\Omega} \gamma\left|\nabla\left(u_{1}-u_{0}\right)\right|^{2}=\int_{D}\left(\gamma_{0}-\gamma_{1}\right) \nabla\left(\bar{u}_{1}-\bar{u}_{0}\right) \nabla u_{0} .
$$

\subsection{The constant case}

Proposition 3.3. Assume $\gamma_{0}$ and $\gamma_{1}$ satisfy (H3i) and let $u_{0}$ and $u_{1}$ solve $(2.3)$ and (2.2). Then

$$
\frac{c_{0}}{\left(c_{0}+\left|\gamma_{1}-\gamma_{0}\right|\right)\left|\gamma_{1}-\gamma_{0}\right|}|\delta W| \leq \int_{D}\left|\nabla u_{0}\right|^{2} \leq\left(\frac{1}{c_{0}}+\frac{2}{\left|\gamma_{1}-\gamma_{0}\right|}\right)|\delta W| .
$$

Proof. Since $\gamma_{0}$ is constant and not zero we can write

$$
\begin{aligned}
& \int_{\Omega} \Im\left(\nabla u_{1} \cdot \nabla \bar{u}_{0}\right)=-\int_{\Omega} \Im\left(\nabla u_{0} \cdot \nabla \bar{u}_{1}-\nabla u_{0} \cdot \nabla \bar{u}_{0}\right) \\
& =-\int_{\Omega} \Im\left(\gamma_{0}\left(\nabla u_{0} \cdot \nabla \bar{u}_{1}-\nabla u_{0} \cdot \nabla \bar{u}_{0}\right) \frac{1}{\gamma_{0}}\right) \\
& =-\Im\left(\frac{1}{\gamma_{0}} \int_{\Omega} \gamma_{0}\left(\nabla u_{0} \cdot \nabla \bar{u}_{1}-\nabla u_{0} \cdot \nabla \bar{u}_{0}\right)\right)=-\Im\left(\frac{1}{\gamma_{0}} \int_{\partial \Omega} h\left(\bar{u}_{1}-\bar{u}_{0}\right)\right)=-\Im\left(\frac{\delta W}{\gamma_{0}}\right),
\end{aligned}
$$

and, hence,

$$
\int_{\Omega} \gamma_{0} \Im\left(\nabla u_{1} \cdot \nabla \bar{u}_{0}\right)=-\gamma_{0} \Im\left(\frac{\delta W}{\gamma_{0}}\right) .
$$

Then, if we set

$$
\delta V=\delta W-2 i \gamma_{0} \Im\left(\frac{\delta W}{\gamma_{0}}\right)=\delta W+2 i \int_{\Omega} \gamma_{0} \Im\left(\nabla u_{1} \cdot \nabla \bar{u}_{0}\right)
$$

we can write the identities of Lemma 3.1 as

$$
\begin{array}{ll}
\text { (id1c) } & \int_{\Omega} \gamma\left|\nabla\left(u_{1}-u_{0}\right)\right|^{2}-\int_{D}\left(\gamma_{1}-\gamma_{0}\right)\left|\nabla u_{0}\right|^{2}=2 i \int_{D}\left(\gamma_{1}-\gamma_{0}\right) \Im\left(\nabla u_{1} \cdot \nabla \bar{u}_{0}\right)+\delta V, \\
(\text { id2c }) & \int_{\Omega} \gamma_{0}\left|\nabla\left(u_{1}-u_{0}\right)\right|^{2}+\int_{D}\left(\gamma_{1}-\gamma_{0}\right)\left|\nabla u_{1}\right|^{2}=-\delta V, \\
(\text { id3c }) & \int_{D}\left(\gamma_{0}-\gamma_{1}\right) \nabla u_{1} \cdot \nabla \bar{u}_{0}=\delta V, \\
(\text { id4c }) & \int_{D}\left(\gamma_{1}-\gamma_{0}\right) \nabla u_{0} \cdot \nabla \bar{u}_{1}=-2 i \int_{D}\left(\gamma_{1}-\gamma_{0}\right) \Im\left(\nabla u_{1} \cdot \nabla \bar{u}_{0}\right)-\delta V .
\end{array}
$$


We write

$$
\begin{aligned}
\int_{D}\left|\nabla u_{0}\right|^{2} & =\int_{D}\left|\nabla\left(u_{0}-u_{1}\right)\right|^{2}-\int_{D}\left|\nabla u_{1}\right|^{2}+2 \int_{D} \Re\left(\nabla u_{1} \cdot \nabla \bar{u}_{0}\right) \\
& \leq \int_{\Omega}\left|\nabla\left(u_{0}-u_{1}\right)\right|^{2}-\int_{D}\left|\nabla u_{1}\right|^{2}+2 \int_{D} \Re\left(\nabla u_{1} \cdot \nabla \bar{u}_{0}\right) .
\end{aligned}
$$

By taking the real part of (id2c) we get

$$
\int_{\Omega} \sigma_{0}\left|\nabla\left(u_{0}-u_{1}\right)\right|^{2}+\left(\sigma_{1}-\sigma_{0}\right) \int_{D}\left|\nabla u_{1}\right|^{2}=-\Re(\delta V) .
$$

By dividing by the positive constant $\sigma_{0}$ and using the fact that both $\sigma_{0}$ and $\sigma_{1}$ are positive we have

$$
\int_{\Omega}\left|\nabla\left(u_{0}-u_{1}\right)\right|^{2}-\int_{D}\left|\nabla u_{1}\right|^{2} \leq-\frac{\Re(\delta V)}{\sigma_{0}} .
$$

Now we divide $(\mathbf{i d 3 c})$ by the constant $\gamma_{0}-\gamma_{1} \neq 0$ and take the real part. We get

$$
\int_{D} \Re\left(\nabla u_{1} \cdot \nabla \bar{u}_{0}\right)=\Re\left(\frac{\delta V}{\gamma_{0}-\gamma_{1}}\right),
$$

which, together with (3.7) and (3.6), gives

$$
\int_{D}\left|\nabla u_{0}\right|^{2} \leq-\frac{\Re(\delta V)}{\sigma_{0}}+2 \Re\left(\frac{\delta V}{\gamma_{0}-\gamma_{1}}\right) .
$$

This leads to the upper bound

$$
\int_{D}\left|\nabla u_{0}\right|^{2} \leq|\delta V|\left(\frac{1}{c_{0}}+\frac{2}{\left|\gamma_{0}-\gamma_{1}\right|}\right) .
$$

To prove the lower bound observe that, by (3.4) and since $\Re \gamma \geq c_{0}$, we have

$$
\left(\int_{\Omega}\left|\nabla\left(u_{0}-u_{1}\right)\right|^{2}\right)^{1 / 2} \leq \frac{\left|\gamma_{0}-\gamma_{1}\right|}{c_{0}}\left(\int_{D}\left|\nabla u_{0}\right|^{2}\right)^{1 / 2} .
$$

Hence, using the identity (id3c), we have

$$
\begin{aligned}
|\delta V| & =\left|\int_{D}\left(\gamma_{0}-\gamma_{1}\right) \nabla u_{1} \cdot \nabla \bar{u}_{0}\right|=\left|\left(\gamma_{0}-\gamma_{1}\right)\left(\int_{D} \nabla\left(u_{1}-u_{0}\right) \cdot \nabla \bar{u}_{0}+\int_{D}\left|\nabla u_{0}\right|^{2}\right)\right| \\
& \leq\left|\gamma_{0}-\gamma_{1}\right|\left(\left(\int_{D}\left|\nabla\left(u_{1}-u_{0}\right)\right|^{2}\right)^{1 / 2}\left(\int_{D}\left|\nabla u_{0}\right|\right)^{1 / 2}+\int_{D}\left|\nabla u_{0}\right|^{2}\right) \\
& \leq\left|\gamma_{0}-\gamma_{1}\right|\left(\frac{\left|\gamma_{0}-\gamma_{1}\right|}{c_{0}} \int_{D}\left|\nabla u_{0}\right|^{2}+\int_{D}\left|\nabla u_{0}\right|^{2}\right),
\end{aligned}
$$

from which the lower bound

$$
\int_{D}\left|\nabla u_{0}\right|^{2} \geq \frac{1}{\left|\gamma_{0}-\gamma_{1}\right|\left(\left|\gamma_{0}-\gamma_{1}\right| / c_{0}+1\right)}|\delta V|
$$

follows. 
Now, by using (3.5), we can see that

$$
\delta V=\frac{\gamma_{0}^{2}}{\left|\gamma_{0}\right|^{2}} \overline{\delta W}
$$

Hence, in particular,

$$
|\delta V|=|\delta W|
$$

and the claim follows.

\subsection{The variable case}

Proposition 3.4. Assume $\gamma_{0}$ and $\gamma_{1}$ satisfy (H3ii) and let $u_{0}$ be the solution of (2.3). Then

$$
K_{1}|\delta W| \leq \int_{D}\left|\nabla u_{0}\right|^{2} \leq K_{2}|\delta W|
$$

where

$$
K_{1}=\frac{c_{0}^{3}}{2\left(2+c_{0}^{2}\right)} \quad \text { and } \quad K_{2}=2\left(\frac{1}{\mu_{0} c_{0}^{2}}+\frac{1}{\mu_{0}}+\frac{1}{c_{0}}\right) .
$$

Proof. If (H3ii) holds, then $\gamma_{0}=\sigma_{0}$ and $\epsilon_{0}=0$. In this case, we have

$$
\begin{aligned}
\int_{\Omega} \sigma_{0} \Im\left(\nabla u_{1} \cdot \nabla \bar{u}_{0}\right) & =\int_{\Omega} \sigma_{0} \Im\left(\nabla u_{1} \cdot \nabla \bar{u}_{0}-\nabla u_{0} \cdot \nabla \bar{u}_{0}\right) \\
& =\Im\left(\int_{\Omega} \sigma_{0} \nabla \bar{u}_{0} \cdot \nabla u_{1}-\sigma_{0} \nabla \bar{u}_{0} \cdot \nabla u_{0}\right) \\
& =\Im\left(\int_{\partial \Omega} \bar{h} u_{1}-\int_{\partial \Omega} \bar{h} u_{0}\right)=\Im(\overline{\delta W})=-\Im(\delta W),
\end{aligned}
$$

and the energy identities become

$$
\begin{array}{ll}
\left(\text { id1 }^{*}\right) & \int_{\Omega} \gamma\left|\nabla\left(u_{0}-u_{1}\right)\right|^{2}-\int_{D}\left(\gamma_{1}-\gamma_{0}\right)\left|\nabla u_{0}\right|^{2}=\overline{\delta W}+2 i \int_{D}\left(\gamma_{1}-\gamma_{0}\right) \Im\left(\nabla u_{1} \cdot \nabla \bar{u}_{0}\right), \\
\left(\text { id2 }^{*}\right) & \int_{\Omega} \gamma_{0}\left|\nabla\left(u_{0}-u_{1}\right)\right|^{2}+\int_{D}\left(\gamma_{1}-\gamma_{0}\right)\left|\nabla u_{1}\right|^{2}=-\overline{\delta W}, \\
\left(\text { id3 }^{*}\right) & \int_{D}\left(\gamma_{0}-\gamma_{1}\right) \nabla u_{1} \cdot \nabla \bar{u}_{0}=\overline{\delta W} .
\end{array}
$$

By $\left(\mathbf{i d} 3^{*}\right)$ we have that

$$
\begin{aligned}
|\overline{\delta W}| & =\left|\int_{D}\left(\gamma_{0}-\gamma_{1}\right) \nabla u_{1} \nabla \bar{u}_{0}\right| \\
& =\left.\left|\int_{D}\left(\gamma_{0}-\gamma_{1}\right) \nabla\left(u_{1}-u_{0}\right) \nabla \bar{u}_{0}+\int_{D}\left(\gamma_{0}-\gamma_{1}\right)\right| \nabla u_{0}\right|^{2} \mid \\
(3.10) \quad & \leq \sup _{D}\left|\gamma_{0}-\gamma_{1}\right|\left(\left(\int_{D}\left|\nabla\left(u_{0}-u_{1}\right)\right|^{2}\right)^{1 / 2}\left(\int_{D}\left|\nabla u_{0}\right|^{2}\right)^{1 / 2}+\int_{D}\left|\nabla u_{0}\right|^{2}\right) .
\end{aligned}
$$


By (3.4), we have

$$
\left(\int_{\Omega}\left|\nabla\left(u_{1}-u_{0}\right)\right|^{2}\right)^{1 / 2} \leq \frac{\sup _{D}\left|\gamma_{0}-\gamma_{1}\right|}{c_{0}}\left(\int_{D}\left|\nabla u_{0}\right|^{2}\right)^{1 / 2},
$$

and by combining this with (3.10) we get

$$
|\delta W| \leq \sup _{D}\left|\gamma_{0}-\gamma_{1}\right|\left(\frac{\sup _{D}\left|\gamma_{0}-\gamma_{1}\right|}{c_{0}}+1\right) \int_{D}\left|\nabla u_{0}\right|^{2} \leq \frac{2}{c_{0}}\left(\frac{2}{c_{0}^{2}}+1\right) \int_{D}\left|\nabla u_{0}\right|^{2},
$$

and one side of the estimate (3.9) follows.

To derive the upper bound, let us first assume

$$
\sigma_{1}-\sigma_{0} \geq \mu_{0}
$$

From the real part of (id2*) we get

$$
\int_{\Omega} \sigma_{0}\left|\nabla\left(u_{1}-u_{0}\right)\right|^{2}+\int_{D}\left(\sigma_{1}-\sigma_{0}\right)\left|\nabla u_{1}\right|^{2}=-\Re(\delta W),
$$

hence, by assumption (3.11),

$$
\begin{aligned}
& \int_{\Omega}\left|\nabla\left(u_{1}-u_{0}\right)\right|^{2} \leq-\frac{\Re(\delta W)}{c_{0}}, \\
& \int_{D}\left|\nabla u_{1}\right|^{2} \leq-\frac{\Re(\delta W)}{\mu_{0}}, \\
& \int_{D}\left|\nabla u_{0}\right|^{2} \leq 2 \int_{D}\left|\nabla\left(u_{0}-u_{1}\right)\right|^{2}+2 \int_{D}\left|\nabla u_{1}\right|^{2} \leq-2\left(\frac{1}{c_{0}}+\frac{1}{\mu_{0}}\right) \Re(\delta W) .
\end{aligned}
$$

On the other hand, if $\left|\epsilon_{1}\right| \geq \mu_{0}$, then, from the imaginary part of (id2*), we get

$$
\int_{D}\left|\epsilon_{1}\right|\left|\nabla u_{1}\right|^{2}=|\Im(\delta W)|
$$

and, hence,

$$
\int_{D}\left|\nabla u_{1}\right|^{2} \leq \frac{|\Im(\delta W)|}{\mu_{0}}
$$

From the real part of (id2*) (see (3.12)) and from (3.13) we get

$$
\begin{aligned}
\int_{\Omega}\left|\nabla\left(u_{1}-u_{0}\right)\right|^{2} & \leq \int_{\Omega} \sigma_{0} c_{0}^{-1}\left|\nabla\left(u_{1}-u_{0}\right)\right|^{2}=c_{0}^{-1} \int_{D}\left(\sigma_{0}-\sigma_{1}\right)\left|\nabla u_{1}\right|^{2}-c_{0}^{-1} \Re(\delta W) \\
& \leq c_{0}^{-1} \sup _{D}\left|\sigma_{0}-\sigma_{1}\right| \int_{D}\left|\nabla u_{1}\right|^{2}-c_{0}^{-1} \Re(\delta W) \\
& \leq \frac{1}{c_{0} \mu_{0}} \sup _{D}\left|\sigma_{0}-\sigma_{1}\right||\Im(\delta W)|-c_{0}^{-1} \Re(\delta W) \\
& \leq \frac{1}{c_{0}^{2} \mu_{0}}|\Im(\delta W)|-\frac{1}{c_{0}} \Re(\delta W) .
\end{aligned}
$$

By (3.13) and (3.14) we get the upper bound

$$
\int_{D}\left|\nabla u_{0}\right|^{2} \leq 2\left(\frac{1}{\mu_{0} c_{0}^{2}}+\frac{1}{c_{0}}+\frac{1}{\mu_{0}}\right)|\delta W| .
$$




\subsection{A one-dimensional example}

We are not able to derive energy bounds and hence also estimates on the size of $D$ for arbitrary variable admittivities. Although the lack of symmetry in condition (H3ii) may seem unnatural, it is in some sense optimal, as the following example shows.

On the other hand we have seen in Proposition 3.4 that assumption (H3ii) leads to energy estimates. The lack of symmetry of condition (H3ii), that seems not natural, is in some sense optimal as the following example shows.

Let $\Omega=(-1,1)$ and let $D=[a, b] \subset(-1,1)$. Consider the background solution $u_{0}$ of

$$
\left\{\begin{aligned}
\left(\gamma_{0} u_{0}^{\prime}\right)^{\prime} & =0 \text { in }(-1,1), \\
\left(\gamma_{0} u_{0}^{\prime}\right)(-1) & =\left(\gamma_{0} u_{0}^{\prime}\right)(1)=K \in \mathbb{C}, \quad u_{0}(-1)+u_{0}(1)=0 .
\end{aligned}\right.
$$

Integrating the equation, $\left(\gamma_{0} u_{0}^{\prime}\right)^{\prime}=0$, and using the normalization conditions one gets that

$$
u_{0}(x)=F_{0}(x)+M, \quad \text { for } x \in(-1,1),
$$

where

$$
F_{0}(x)=\int \frac{K}{\gamma_{0}(x)} d x
$$

and $M=\left(F_{0}(1)+F_{0}(-1)\right) / 2$.

Considering the perturbed solution $u_{1}$ of

$$
\left\{\begin{aligned}
\left(\gamma u_{1}^{\prime}\right)^{\prime} & =0 \text { in }(-1,1), \\
\left(\gamma u_{1}^{\prime}\right)(-1) & =\left(\gamma u_{1}^{\prime}\right)(1)=K \in \mathbb{C}, \quad u_{1}(-1)+u_{1}(1)=0
\end{aligned}\right.
$$

one gets

$$
u_{1}(x)= \begin{cases}F_{0}(x)+M & \text { if } x \in(-1, a), \\ F_{1}(x)+M+\frac{F_{0}(a)+F_{0}(b)}{2}-\frac{F_{1}(a)+F_{1}(b)}{2} & \text { if } x \in(a, b), \\ F_{0}(x)+M+\frac{F_{1}(b)-F_{1}(a)}{2}-\frac{F_{0}(b)-F_{0}(a)}{2} & \text { if } x \in(b, 1),\end{cases}
$$

where

$$
F_{1}(x)=\int \frac{K}{\gamma_{1}(x)} d x
$$

Hence

$$
\begin{aligned}
\delta W & =K\left(\overline{\left.u_{1}(1)-u_{0}(1)\right)-\left(u_{1}(-1)-u_{0}(-1)\right.}\right)=K\left(\overline{u_{1}(1)-u_{0}(1)}\right) \\
& =\frac{|K|^{2}}{2} \int_{a}^{b}\left(\overline{\left.\frac{1}{\gamma_{1}}-\frac{1}{\gamma_{0}}\right)} d x\right. \\
\Re(\delta W) & =\frac{|K|^{2}}{2} \int_{a}^{b}\left(\frac{\sigma_{0}}{\sigma_{0}^{2}+\epsilon_{0}^{2}}-\frac{\sigma_{1}}{\sigma_{1}^{2}+\epsilon_{1}^{2}}\right) d x,
\end{aligned}
$$


and

$$
\Im(\delta W)=\frac{|K|^{2}}{2} \int_{a}^{b}\left(-\frac{\epsilon_{0}}{\sigma_{0}^{2}+\epsilon_{0}^{2}}+\frac{\epsilon_{1}}{\sigma_{1}^{2}+\epsilon_{1}^{2}}\right) d x .
$$

So, if one of the monotonicity conditions

$$
\frac{\sigma_{1}}{\sigma_{1}^{2}+\epsilon_{1}^{2}}>(<) \frac{\sigma_{0}}{\sigma_{0}^{2}+\epsilon_{0}^{2}} \text { in }(-1,1)
$$

or

$$
\frac{\epsilon_{1}}{\sigma_{1}^{2}+\epsilon_{1}^{2}}>(<) \frac{\epsilon_{0}}{\sigma_{0}^{2}+\epsilon_{0}^{2}} \text { in }(-1,1)
$$

holds, then either $\Re(\delta W) \neq 0$ or $\Im(\delta W) \neq 0$ and $\delta W$ recovers $(a, b)$ uniquely.

In particular observe that if $\epsilon_{0}=0$ we find that $\Im(\delta W) \neq 0$ if $\epsilon_{1}$ has constant sign in $(-1,1)$ and $\Re(\delta W) \neq 0$ if $\sigma_{1}-\sigma_{0}>0$ in $(-1,1)$ which are exactly the condition (H3ii). If the above conditions fail uniqueness does not hold. Consider, for example, $\gamma_{0}=(2+i x)^{2}$ for $x \in(-1,1)$ and $\gamma_{1}=17 / 4$. Then one easily sees that

$$
\begin{aligned}
& \Re(\delta W)=|K|^{2}(b-a)\left(\frac{4}{17}-\frac{4-a b}{\left(4+b^{2}\right)\left(4+a^{2}\right)}\right), \\
& \Im(\delta W)=|K|^{2}(b-a)\left(-\frac{2(a+b)}{\left(4+b^{2}\right)\left(4+a^{2}\right)}\right)
\end{aligned}
$$

and clearly $\Re(\delta W)=\Im(\delta W)=0$ for $a=1 / 2$ and $b=-1 / 2$.

\section{Main tools: quantitative estimates of unique continuation}

We list now various forms of the quantitative estimates of unique continuation that we will need in the sequel. Throughout this section we will assume that $\Omega \subset \mathbb{R}^{n}$ is a bounded domain of class $C^{0,1}$ with constants $r_{0}$ and $M_{0}$. and $A$ is a symmetric $n \times n$ matrix with real entries defined in $\mathbb{R}^{n}$ satisfying:

(Uniform ellipticity) For a given $\lambda_{0}, 0<\lambda_{0} \leq 1$,

$$
\lambda_{0}|\xi|^{2} \leq A(x) \xi \cdot \xi \leq \lambda_{0}{ }^{-1}|\xi|^{2}, \text { for every } \xi \in \mathbb{R}^{n}, x \in \mathbb{R}^{n} .
$$

(Lipschitz regularity) For a given $\lambda_{1}>0$,

$$
|A(x)-A(y)| \leq \frac{\lambda_{1}}{r_{0}}|x-y|, \text { for every } x, y \in \mathbb{R}^{n} .
$$

Theorem 4.1 (Three spheres inequality, [3]). Let $u \in H^{1}(\Omega)$ be a solution to the equation

$$
\operatorname{div}(A(x) \nabla u(x))=0 \quad \text { in } \Omega .
$$

For every $r_{1}, r_{2}, r_{3}, \bar{r}, 0<r_{1}<r_{2}<r_{3} \leq \bar{r}$, and for every $x_{0} \in \Omega_{\bar{r}}$,

$$
\int_{B_{r_{2}}\left(x_{0}\right)}\left|\nabla u_{0}\right|^{2} \leq C\left(\int_{B_{r_{1}}\left(x_{0}\right)}|\nabla u|^{2}\right)^{\theta}\left(\int_{B_{r_{3}}\left(x_{0}\right)}|\nabla u|^{2}\right)^{1-\theta},
$$

where $C>0$ and $\theta, 0<\theta<1$, only depend on $\lambda_{0}, \lambda_{1}, r_{1} / r_{3}$, and $r_{2} / r_{3}$. 
Theorem 4.2 (Lipschitz propagation of smallness, [3]). Let $h$ satisfy (H4) and let $u \in H^{1}(\Omega)$ be the solution of the Neumann problem

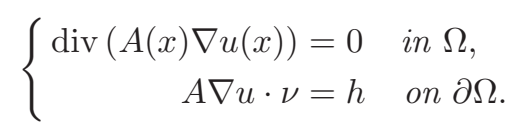

For every $\rho>0$ and for every $x \in \Omega_{2}$, we have

$$
\int_{B_{\rho}(x)}|\nabla u|^{2} \geq C^{-1} \int_{\Omega}|\nabla u|^{2}
$$

where $C \geq 1$ only depends on $\lambda_{0}, \lambda_{1}, M_{0}, M_{1}, F(h)$, and $\rho / r_{0}$.

The three spheres inequality and the Lipschitz propagation of smallness in [3] are obtained for real valued functions $u$ and $h$ but with straightforward modifications they apply to complex valued functions.

Theorem 4.3 (Doubling inequality). Let $u \in H^{1}\left(B_{r_{0}}\left(x_{0}\right)\right)$ be the solution of

$$
\operatorname{div}(A(x) \nabla u(x))=0 \quad \text { in } B_{r_{0}}\left(x_{0}\right) .
$$

Then, there exist positive constants $\alpha$ and $C$, depending only on $\lambda_{0}$ and on $\lambda_{1}$, such that

$$
\int_{B_{2 r}\left(x_{0}\right)}|\nabla u|^{2} \leq C\left(\frac{\int_{B_{r_{0}}\left(x_{0}\right)}|\nabla u|^{2}}{\int_{B_{r_{0} / 2}\left(x_{0}\right)}|\nabla u|^{2}}\right)^{\alpha} \int_{B_{r}\left(x_{0}\right)}|\nabla u|^{2},
$$

for every $r$ such that $0<r \leq r_{0} / 2$.

The doubling inequality was first derived by Garofalo and Lin in [15]. Later it was also derived by Kukavica in [20] using Rellich's identity. In the appendix, for the convenience of the reader, we will give the proof of the doubling inequality following the proof in [20], showing the modifications one must make in the case of complex-valued functions and estimating more carefully the constant occurring in the inequality.

Lemma 4.4. Let $\Omega$ satisfy (H1), let $\bar{r}$ and $R$ be positive numbers such that $3 \sqrt{n} R<\bar{r}$, and let $u \in H^{1}(\Omega)$ be a nontrivial solution of

$$
\operatorname{div}(A(x) \nabla u(x))=0 \quad \text { in } \Omega .
$$

Assume that $\Omega_{\bar{r}} \neq \emptyset$. Then, for every $x_{0} \in \Omega_{\bar{r}}$ and for every measurable set $E \subset Q_{R}\left(x_{0}\right)$, we have

$$
\frac{|E|}{\left|Q_{R}\left(x_{0}\right)\right|} \leq\left(\frac{H \int_{E}|\nabla u|^{2}}{\int_{Q_{R}\left(x_{0}\right)}|\nabla u|^{2}}\right)^{1 / p}
$$


where $H$ and $p>1$ are given by

$$
p=1+\frac{\log 4 F_{\bar{r}}(u)}{\log (17 / 16)}, \quad \text { and } \quad H=\left(27 F_{\bar{r}}(u)\right)^{p(p-1)},
$$

where

$$
F_{\bar{r}}(u)=C\left(\frac{\int_{\Omega}|\nabla u|^{2}}{\int_{\Omega_{\bar{r} / 2}}|\nabla u|^{2}}\right)^{C}
$$

and $C$ depends on $\lambda_{0}, \lambda_{1}, M_{0}, M_{1}$, and $\bar{r} / r_{0}$.

Sketch of the proof. Lemma 4.4 can be proved by adapting the proof of Lemma 2.4 in [21]. We describe in detail the necessary changes. The most important difference between Lemma 2.4 in [21] and our lemma is that $|\nabla u|^{2}$ appears in the bound (4.6) while in [21] $|u|^{2}$ is involved.

Observe that $|\nabla u|^{2}$ satisfies the following reverse Hölder inequality (RHI):

$$
\begin{aligned}
\left(\frac{1}{\left|Q_{R}\left(x_{0}\right)\right|} \int_{Q_{R}\left(x_{0}\right)}\right. & \left.\left(|\nabla u|^{2}\right)^{1+\delta}\right)^{1 /(1+\delta)} \\
& \leq \frac{C}{\left|Q_{R}\left(x_{0}\right)\right|}\left(\frac{\left.\int_{B_{\bar{r}\left(x_{0}\right)}|\nabla u|^{2}}\right)^{\alpha} \int_{B_{\bar{r} / 2}\left(x_{0}\right)}|\nabla u|^{2}}{\int_{Q_{R}\left(x_{0}\right)}|\nabla u|^{2}}\right.
\end{aligned}
$$

for any $x_{0} \in \Omega_{\bar{r}}$ and $R$ such that $0<2 \sqrt{n} R \leq \bar{r}$, where $C$ and $\alpha$ depend only on $\lambda_{0}$ and $\lambda_{1}$ and $\delta>0$ is arbitrary.

In fact, if we set

$$
\tau=\frac{1}{\left|Q_{R}\left(x_{0}\right)\right|} \int_{Q_{R}\left(x_{0}\right)} u(x) d x,
$$

from [16], the Poincaré inequality and (4.5) we get,

$$
\begin{aligned}
\sup _{Q_{R}\left(x_{0}\right)}|\nabla u|^{2} & \leq \frac{C}{R^{2}} \sup _{Q_{3 / 2 R}\left(x_{0}\right)}|u-\tau|^{2} \leq \frac{C^{\prime}}{R^{n+2}} \int_{Q_{2 R}\left(x_{0}\right)}|u-\tau|^{2} \\
& \leq \frac{C^{\prime \prime}}{R^{n}} \int_{Q_{2 R}\left(x_{0}\right)}|\nabla u|^{2} \leq \frac{C^{\prime \prime}}{R^{n}} \int_{B_{2 \sqrt{n} R}\left(x_{0}\right)}|\nabla u|^{2} \\
& \leq \frac{C^{\prime \prime \prime}}{R^{n}}\left(\frac{\int_{B_{\bar{r}}\left(x_{0}\right)}|\nabla u|^{2}}{\int_{B_{\bar{r} / 2}\left(x_{0}\right)}|\nabla u|^{2}}\right)^{\alpha} \int_{Q_{R}\left(x_{0}\right)}|\nabla u|^{2},
\end{aligned}
$$

where $C^{\prime}, C^{\prime \prime}$ and $C^{\prime \prime \prime}$ and $\alpha$ depend on $\lambda_{0}$ and $\lambda_{1}$ only. We derive (4.8) in a trivial manner.

Using iteratively the three spheres inequality we get the estimate (see [4])

$$
\int_{\Omega_{\bar{r} / 2}}|\nabla u|^{2} \leq C\left(\int_{B_{\bar{r} / 2}\left(x_{0}\right)}|\nabla u|^{2}\right)^{\theta}\left(\int_{\Omega}|\nabla u|^{2}\right)^{1-\theta},
$$


where $0<\theta<1$ and $\theta$ and $C$ depend on $\lambda_{0}, \lambda_{1}, M_{0}, M_{1}$ and $\bar{r} / r_{0}$. From (4.9) we have trivially

$$
\frac{\int_{B_{\bar{r}}\left(x_{0}\right)}|\nabla u|^{2}}{\int_{B_{\bar{r} / 2}\left(x_{0}\right)}|\nabla u|^{2}} \leq \frac{\int_{\Omega}|\nabla u|^{2}}{\int_{B_{\bar{r} / 2}\left(x_{0}\right)}|\nabla u|^{2}} \leq\left(\frac{C \int_{\Omega}|\nabla u|^{2}}{\int_{\Omega_{\bar{r} / 2}}|\nabla u|^{2}}\right)^{1 / \theta} .
$$

From (4.10) and (4.8) we get the following version of RHI:

$$
\left(\frac{1}{\left|Q_{R}\left(x_{0}\right)\right|} \int_{\mid Q_{R}\left(x_{0}\right)}\left(|\nabla u|^{2}\right)^{1+\delta}\right)^{1 /(1+\delta)} \leq \frac{F}{\left|Q_{R}\left(x_{0}\right)\right|} \int_{Q_{R}\left(x_{0}\right)}|\nabla u|^{2},
$$

for any $x_{0} \in \Omega_{\bar{r}}$ and for any $R$ such that $R \in\left(0, \frac{\bar{r}}{2 \sqrt{n}}\right]$ and $\delta>0$, where

$$
F=\left(\frac{C \int_{\Omega}|\nabla u|^{2}}{\int_{\Omega_{\bar{r} / 2}}|\nabla u|^{2}}\right)^{\alpha / \theta}
$$

with $C, \alpha$ and $\theta$ depending only on $\lambda_{0}, \lambda_{1}, M_{0}, M_{1}$, and $\bar{r} / r_{0}$. In order to prove (4.9) we used the Lipschitz regularity of $\partial \Omega$ in order to guarantee that $\Omega_{\rho}$ is a connected set for $\rho$ sufficiently small. If (4.11) holds for $\bar{r}$ small then it clearly holds also for large $\bar{r}$. The most difficult part of the proof is to show that the lemma follows from (4.11) but this can be found in Theorem 2.11 in [14], while an explicit evaluation of the constants can be found in [21].

\section{Proof of the main results}

In this section we will use the quantitative unique continuation estimates stated in the previous section and regularity results for solutions of elliptic equations to get upper and lower bounds of the measure $|D|$ of the inclusion $D$, in terms of the energy related to the background potential $u_{0}$.

Throughout this section we will assume that $A$ is a symmetric real $n \times n$ matrix defined in $\mathbb{R}^{n}$ satisfying (4.1) and (4.2)

Proposition 5.1. Let $\Omega \subset \mathbb{R}^{n}$ satisfy (H1) with $\partial \Omega$ of class $C^{0,1}$ and let $D \subset \Omega$ satisfy $(\mathbf{H 2 a})$. Let $h$ satisfy $(\mathbf{H} 4 \mathbf{a})$ and let $u \in H^{1}(\Omega)$ be a solution to the Neumann problem (4.3) such that

$$
\int_{\partial \Omega} u=0
$$

Then

$$
\left(\frac{|D|}{|\Omega|}\right)^{p} \leq C\left(\frac{\int_{D}|\nabla u|^{2}}{\int_{\Omega}|\nabla u|^{2}}\right)
$$

where $p, p>1$ and $C$ depend only on $d_{0} / r_{0}, M_{0}, M_{1}, \lambda_{0}, \lambda_{1}$, and $F(h)$, where $F(h)$ is given by (2.1). 
Proof. Let $\delta=d_{0} /(4 \sqrt{n})$ and cover $D$ with pairwise internally disjoint closed cubes, $Q_{j}, j=1, \ldots, N$, of side length $\delta$. Assume that $Q_{j} \cap D \neq \emptyset$ for $j=1, \ldots, N$. We have

$$
D \subset \cup_{j=1}^{N} Q_{j} \subset \Omega_{\frac{3}{4} d_{0}} .
$$

The value of $p>1$ will be chosen later. From (5.3) and Hölder inequality (in what follows $\left.p^{\prime}=(p-1) / p\right)$ we get

$$
\begin{aligned}
|D| & =\sum_{j=1}^{N}\left|D \cap Q_{j}\right|=\sum_{j=1}^{N} \frac{\left|D \cap Q_{j}\right|}{\left|Q_{j}\right|}\left|Q_{j}\right| \\
& \leq\left(\sum_{j=1}^{N}\left(\frac{\left|D \cap Q_{j}\right|}{\left|Q_{j}\right|}\right)^{p}\right)^{1 / p}\left(\sum_{j=1}^{N}\left|Q_{j}\right|^{p^{\prime}}\right)^{1 / p^{\prime}} \leq\left|\Omega_{\frac{3}{4} d_{0}}\right|\left(\sum_{j=1}^{N}\left(\frac{\left|D \cap Q_{j}\right|}{\left|Q_{j}\right|}\right)^{p}\right)^{1 / p} .
\end{aligned}
$$

Hence, for any $p>1$ we have

$$
\left(\frac{|D|}{|\Omega|}\right)^{p} \leq \sum_{j=1}^{N}\left(\frac{\left|D \cap Q_{j}\right|}{\left|Q_{j}\right|}\right)^{p}
$$

Now, in order to choose $p$ and to bound the right-hand side of (5.4) we apply Lemma 4.4 with $\bar{r}=\frac{3}{4} d_{0}$ and we bound $F_{\bar{r}}(h)$ defined in (4.7) from above. We bound $\int_{\Omega_{\bar{r} / 2}}|\nabla u|^{2}$ from below by observing that, for $\bar{x} \in \Omega_{\bar{r}}$, applying the Lipschitz propagation of smallness (LPS) with $\rho=\bar{r} / 2$, we get

$$
\int_{\Omega_{\bar{r} / 2}}|\nabla u|^{2} \geq \int_{B_{\bar{r} / 2}(\bar{x})}|\nabla u|^{2} \geq C_{1}^{-1} \int_{\Omega}|\nabla u|^{2},
$$

where $C_{1} \geq 1$ depends on $d_{0} / r_{0}, M_{0}, M_{1}, \lambda_{0}, \lambda_{1}$, and $F(h)$, where $F(h)$ is given by (2.1). Hence, by (5.5), we obtain that

$$
F_{\bar{r}}(h) \leq C_{1} .
$$

Now let

$$
p=1+\frac{\log 4 C_{1}^{2}}{\log (17 / 16)}
$$

By Lemma 4.4 we have

$$
\left(\frac{\left|D \cap Q_{j}\right|}{\left|Q_{j}\right|}\right)^{p} \leq\left(27 C_{1}^{2}\right)^{p(p-1)} \frac{\int_{D \cap Q_{j}}|\nabla u|^{2}}{\int_{Q_{j}}|\nabla u|^{2}}, \quad j=1, \ldots, N .
$$

We use the LPS property again to estimate the right-hand side of (5.7) from above. Denoting by $x_{j}$ the center of the cube $Q_{j}$ we have

$$
\int_{Q_{j}}|\nabla u|^{2} \geq \int_{B_{\delta / 2}\left(x_{j}\right)}|\nabla u|^{2} \geq C_{2}^{-1} \int_{\Omega}|\nabla u|^{2},
$$

where $C_{2} \geq 1$ depends on $d_{0} / r_{0}, M_{0}, M_{1}, \lambda_{0}, \lambda_{1}$, and $F(h)$. By (5.8), (5.7), (5.6), and (5.4) we get the claim. 
Proposition 5.2. Let $\Omega \subset \mathbb{R}^{n}$ satisfy (H1) with $\partial \Omega$ of class $C^{1,1}$, let $D \subset \Omega$

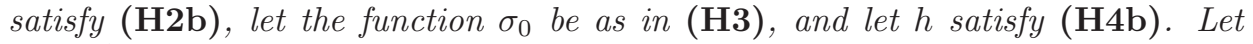
$u \in H^{1}(\Omega)$ be the solution to the Neumann problem

$$
\begin{cases}\operatorname{div}\left(\left(\sigma_{0}(x) \nabla u(x)\right)=0\right. & \text { in } \Omega, \\ \sigma_{0} \nabla u \cdot \nu=h & \text { on } \partial \Omega,\end{cases}
$$

satisfying the normalization condition (5.1). Then

$$
\left(\frac{|D|}{|\Omega|}\right)^{p} \leq C\left(\frac{\int_{D}|\nabla u|^{2}}{\int_{\Omega}|\nabla u|^{2}}\right)
$$

where $p, p>1$ and $C$ depend only on $r_{1} / r_{0}, M_{0}, M_{1}, c_{0}, L$, and $F(h)$, where $F(h)$ is given by (2.1).

Proof. Define $\Gamma:=\partial \Omega \cap \bar{B}_{r_{1}}(P)$. First we construct a suitable family of cylinders covering $\partial \Omega \backslash \Gamma$.

Let

$$
r_{2}=\min \left\{\frac{r_{1}}{4 \sqrt{n}}, \frac{r_{0}}{2 \sqrt{n} M_{0}}\right\}
$$

and fix $r \in\left(0, r_{2}\right]$, to be chosen later. Let $\left\{Q_{j}\right\}_{j=1}^{J}$ a family of closed mutually internally disjoint cubes of side length $2 r$ such that

$$
(\partial \Omega \backslash \Gamma) \bigcap Q_{j} \neq \emptyset, \quad j=1, \ldots, J, \quad \text { and } \quad \partial \Omega \backslash \Gamma \subset \bigcup_{j=1}^{J} Q_{j} .
$$

Fix $j \in\{1, \ldots, J\}$ and let $x_{j} \in(\partial \Omega \backslash \Gamma) \cap Q_{j}$. Let $\nu_{j}$ be the exterior unit normal vector to $\partial \Omega$ at $x_{j}$ on Let $R_{j}$ the cylinder centered at $x_{j}$ with axis parallel to $\nu_{j}$ and with base a ball of radius $2 \sqrt{n} r$ and with height $2 \sqrt{n} M_{0} r$. Setting $\tilde{R}_{j}=$ $2\left(R_{j}-x_{j}\right)+x_{j}$ one sees easily that

$$
\bigcup_{j=1}^{J} \tilde{R}_{j} \supset \Omega \backslash \Omega_{2 \sqrt{n} r}
$$

and hence

$$
\operatorname{dist}\left(\Omega \backslash \bigcup_{j=1}^{J} \tilde{R}_{j}, \partial \Omega\right) \geq 2 \sqrt{n} r .
$$

Furthermore, since the interiors of the cubes $Q_{j}, j=1, \ldots, J$, are pairwise disjoint and since, obviously,

$$
\bigcup_{j=1}^{J} Q_{j} \subset\left\{x \in \mathbb{R}^{n}: \operatorname{dist}(x, \partial \Omega)<2 \sqrt{n} r\right\},
$$

we obtain for $J$ the estimate

$$
J \leq(2 r)^{-n}\left|\bigcup_{j=1}^{J} Q_{j}\right| \leq C\left(\frac{r_{0}}{r}\right)^{n-1},
$$


where $C$ depends only on $M_{0}$ and $M_{1}$. Let

$$
D^{\prime}=D \bigcap\left(\bigcup_{j=1}^{J} \tilde{R}_{j}\right), \quad \text { and } \quad D^{\prime \prime}=D \backslash D^{\prime} .
$$

From (5.9) we have

$$
\operatorname{dist}\left(D^{\prime \prime}, \partial \Omega\right) \geq 2 \sqrt{n} r .
$$

From this last inequality and Proposition 5.1 we get

$$
\left(\frac{\left|D^{\prime \prime}\right|}{|\Omega|}\right)^{p} \leq C_{r}\left(\frac{\int_{D^{\prime \prime}}|\nabla u|^{2}}{\int_{\Omega}|\nabla u|^{2}}\right)
$$

where $C_{r}$ depends only on $r / r_{0}, M_{0}, M_{1}, c_{0}, L$, and $F(h)$.

Let, for a fixed index $j \in\{1, \ldots, J\}$,

$$
D_{j}:=\tilde{R}_{j} \cap D^{\prime} \quad \text { and } \quad \hat{R}_{j}:=2\left(\tilde{R}_{j}-x_{j}\right)+x_{j} .
$$

It is easy to see that if

$$
r \leq \min \left\{\frac{r_{1}}{16 \sqrt{n} \sqrt{1+M_{0}^{2}}}, \frac{r_{2}}{2}\right\}
$$

then

$$
\operatorname{dist}\left(\hat{R}_{j}, \Gamma_{0}\right) \geq \frac{r_{1}}{4}
$$

where we recall that $\Gamma_{0}=\Gamma \cap \overline{B_{r_{1} / 2}(P)}$. Furthermore, up to a rigid transformation such that $x_{j}=0$, we have

$$
\hat{R}_{j} \cap \Omega=\left\{\left(x^{\prime}, x_{n}\right) \in \mathbb{R}^{n}: x_{n}>\psi\left(x^{\prime}\right),\left|x^{\prime}\right| \leq 8 \sqrt{n} r,\left|x_{n}\right| \leq 8 \sqrt{n} M_{0} r\right\},
$$

where

$$
\psi(0)=|\nabla \psi(0)|=0
$$

and

$$
\|\psi\|_{L^{\infty}}+r_{0}\|\nabla \psi\|_{L^{\infty}}+r_{0}^{2}\left\|D^{2} \psi\right\|_{L^{\infty}} \leq M_{0} r_{0} .
$$

Without loss of generality we may assume that $\sigma_{0}(0)=1$. Following the arguments of [1] or [2] we can construct a function $\Psi \in C^{1,1}\left(\overline{B_{\rho_{0}}(0)}, \mathbb{R}^{n}\right)$, where $\rho_{0}=16 \sqrt{n} \sqrt{1+M_{0}^{2}} r$ such that

$$
\begin{gathered}
\Psi\left(x^{\prime}, \psi\left(x^{\prime}\right)\right)=\left(x^{\prime}, 0\right), \quad \forall x^{\prime} \in B_{\rho_{0}}^{\prime}(0), \\
\Psi\left(\hat{R}_{j} \cap \Omega\right) \subset\left\{\left(x^{\prime}, x_{n}\right): x_{n}>0\right\} .
\end{gathered}
$$

Moreover, there exist $C_{1}, C_{2} \geq 1$ depending only on $M_{0}$ such that

$$
\begin{aligned}
C_{1}^{-1}|x-z| & \leq|\Psi(x)-\Psi(z)| \leq C_{1}|x-z|, \quad \forall x, z \in B_{\rho_{0}}(0), \\
C_{2}^{-1} & \leq|\operatorname{det} D \Psi(x)| \leq C_{2}, \quad \forall x \in B_{\rho_{0}}(0),
\end{aligned}
$$


and, setting $A(y)=\left\{a_{i j}(y)\right\}_{i j=1}^{n}$, where

$$
\begin{aligned}
A(y) & =\left|\operatorname{det} D \Psi^{-1}(x)\right|(D \Psi)\left(\Psi^{-1}(y)\right) \sigma_{0}\left(\Psi^{-1}(y)\right)(D \Psi)^{T}\left(\Psi^{-1}(y)\right), \\
v(y) & =u\left(\Psi^{-1}(y)\right),
\end{aligned}
$$

we have

$$
\begin{aligned}
& A(y)=\mathrm{Id}, \\
& a_{n k}\left(y^{\prime}, 0\right)=a_{k n}\left(y^{\prime}, 0\right)=0, \quad k=1, \ldots, n, \\
& C_{3}^{-1}|\xi|^{2} \leq A(y) \xi \cdot \xi \leq C_{3}|\xi|^{2}, \quad \forall \xi \in \mathbb{R}^{n}, \forall y \in \Psi\left(\Omega \cap \hat{R}_{j}\right), \\
& |A(y)-A(z)| \leq \frac{C_{4}}{r}|y-z|, \quad \forall y, z \in \Psi\left(\Omega \cap \hat{R}_{j}\right),
\end{aligned}
$$

where, in (5.19), Id denotes the identity matrix and $C_{3}, C_{4} \geq 1$ depend only on $M_{0}$. Furthermore, recalling $\hat{R}_{j} \cap \Gamma_{0} \neq \emptyset$ and (5.12) we have

$$
\begin{cases}\operatorname{div}\left(\left(A(y) \nabla_{y} v(y)\right)=0\right. & \text { in } \Psi\left(\Omega \cap \hat{R}_{j}\right), \\ \frac{\partial v}{\partial y_{n}}\left(y^{\prime}, 0\right)=0 & \text { on } \Psi\left(\partial \Omega \cap \hat{R}_{j}\right) .\end{cases}
$$

From the properties of the matrix $A$, in particular from (5.20), we have that the function $\tilde{v}$ defined by

$$
\tilde{v}\left(y^{\prime}, y_{n}\right):=v\left(y^{\prime},\left|y_{n}\right|\right)
$$

solves an elliptic equation with Lipschitz coefficients in the principal part. More precisely, let $\tilde{A}(y)=\left\{\tilde{a}_{i j}(y)\right\}_{i, j=1}^{n}$ be the matrix with entries

$$
\begin{aligned}
\tilde{a}_{i j}\left(y^{\prime},\left|y_{n}\right|\right) & =a_{i j}\left(y^{\prime},\left|y_{n}\right|\right), \quad \text { if } i, j \in\{1, \ldots, n-1\} \text { or } i=j=n, \\
\tilde{a}_{i j}\left(y^{\prime}, y_{n}\right) & =\tilde{a}_{i j}\left(y^{\prime}, y_{n}\right)=\operatorname{sgn}\left(y_{n}\right) a_{n j}\left(y^{\prime},\left|y_{n}\right|\right), \text { if } i, j \in\{1, \ldots, n-1\} \text { or } i=j .
\end{aligned}
$$

Then we have

$$
\operatorname{div}\left(\left(A(y) \nabla_{y} \tilde{v}(y)\right)=0 \quad \text { in } \hat{\Lambda}_{j}\right.
$$

where

$$
\hat{\Lambda}_{j}=\left\{\left(y^{\prime}, y_{n}\right) \in \mathbb{R}^{n}:\left(y^{\prime},\left|y_{n}\right|\right) \in \hat{\Lambda}_{j}^{+}\right\}
$$

with

$$
\hat{\Lambda}_{j}^{+}=\Psi\left(\Omega \cap \hat{R}_{j}\right) .
$$

It is easy to see that the matrix $\tilde{A}$ satisfies uniform ellipticcity and Lipschitz continuity with the same constants as in (5.21) and (5.22).

In the sequel we will use the notation

$$
\begin{aligned}
\tilde{\Lambda}_{j}^{+} & :=\Psi\left(\Omega \cap \tilde{R}_{j}\right), \\
\tilde{\Lambda}_{j} & :=\left\{\left(y^{\prime}, y_{n}\right) \in \mathbb{R}^{n}:\left(y^{\prime},\left|y_{n}\right|\right) \in \tilde{\Lambda}_{j}^{+}\right\}, \\
\tilde{D}_{j} & :=\Psi\left(D_{j}\right) .
\end{aligned}
$$

Since our aim is to bound $\left|D_{j}\right|$, we proceed initially as in the proof of Proposition 5.1. 
First we note that from (5.15) we get

$$
\operatorname{dist}\left(\tilde{D}_{j}, \partial \hat{\Lambda}_{j}\right) \geq \delta_{0}:=\frac{2 \sqrt{n} r}{C_{1}},
$$

where $C_{1}$ is the constant appearing in (5.15). Cover $\tilde{D}_{j}$ by pairwise internally disjoint closed cubes, $Q_{j, k}, k=1, \ldots, N_{j}$, of side length $\delta_{1}:=\delta_{0} /(4 \sqrt{n})$. We have

$$
\tilde{D}_{j} \subset \bigcup_{k=1}^{N_{j}} Q_{j, k} \subset \hat{\Lambda}_{\frac{3}{4} \delta_{0}} .
$$

Since we are interested in applying Lemma 4.4 with $\Omega=\hat{\Lambda}_{j}$ and $\bar{r}=\delta_{0} / 4$ we need to prove first the following claim

Claim 1. There exists a constant $C$ depending only on $c_{0}, L, M_{0}, M_{1}, r / r_{0}$, and $F(h)$ such that

$$
\tilde{F}_{j, \bar{r}}(\tilde{v}):=\frac{\int_{\hat{\Lambda}_{j}}|\nabla \tilde{v}|^{2}}{\int_{\hat{\Lambda}_{j, \bar{r} / 2}}|\nabla \tilde{v}|^{2}} \leq C, \quad j=1, \ldots, J
$$

(with $C$ independent of $j$ ).

Proof of the claim. Since for $\bar{r}=\delta_{0} / 4$ we have that $\hat{\Lambda}_{j, \bar{r} / 2} \supset \tilde{\Lambda}$, recalling that $\tilde{v}$ is the even reflection of $v=u \circ \Psi^{-1}$, by a change of variables we derive

$$
\tilde{F}_{j, \bar{r}}(\tilde{v}) \leq C \frac{\int_{\hat{R}_{j} \cap \Omega}|\nabla u|^{2}}{\int_{\tilde{R}_{j, \bar{r} / 2} \cap \Omega}|\nabla u|^{2}}, \quad j=1, \ldots, J,
$$

where $C$ depends only on $c_{0}, L, M_{0}, M_{1}$, and $r / r_{0}$. Now, since

$$
\begin{aligned}
& \tilde{R}_{j} \cap \Omega \supset B_{\sqrt{n} r}\left(x_{j}-2 \sqrt{n} r \nu\right):=B^{(j)}, \\
& \operatorname{dist}\left(B^{(j)}, \partial\left(\tilde{R}_{j} \cap \Omega\right)\right) \geq \sqrt{n} r,
\end{aligned}
$$

estimating the right-hand side of (5.26) and applying the LPS property we get

$$
\tilde{F}_{j, \bar{r}}(\tilde{v}) \leq C \frac{\int_{\Omega}|\nabla u|^{2}}{\int_{B^{(j)}}|\nabla u|^{2}} \leq C^{\prime},
$$

where $C^{\prime}$ depends only on $c_{0}, L, M_{0}, M_{1}, r / r_{0}$, and $F(h)$.

We choose $r=r_{2}$. Proceeding as in the proof of Proposition 5.1 and using (5.26) we obtain

$$
\left|\tilde{D}_{j}\right| \leq\left|\hat{\Lambda}_{j}\right|\left(\frac{\int_{\tilde{D}_{j}}|\nabla \tilde{v}|^{2}}{\int_{\hat{\Lambda}_{j}}|\nabla \tilde{v}|^{2}}\right)^{1 / p}, \quad j=1, \ldots, J,
$$

where $C$ and $p \in(1, \infty)$ depend on $c_{0}, L, M_{0}, M_{1}, r_{1} / r_{0}$, and $F(h)$. 
From the definitions of $\tilde{v}$ and of $\hat{\Lambda}_{j}$, with some simple change of variables and using again the LPS property, we derive from (5.27)

$$
\left|D_{j}\right| \leq C|\Omega|\left(\frac{\int_{D_{j}}|\nabla u|^{2}}{\int_{\Omega}|\nabla u|^{2}}\right)^{1 / p}, \quad j=1, \ldots, J,
$$

where $C$ and $p \in(1,+\infty)$ depend on $c_{0}, L, M_{0}, M_{1}, r_{1} / r_{0}$, and $F(h)$.

From (5.28) and from (5.10) we have

$$
\left|D^{\prime}\right| \leq \sum_{j=1}^{J}\left|D_{j}\right| \leq C|\Omega|\left(\frac{\int_{D^{\prime}}|\nabla u|^{2}}{\int_{\Omega}|\nabla u|^{2}}\right)^{1 / p},
$$

where $C$ and $p \in(1, \infty)$ depend on $c_{0}, L, M_{0}, M_{1}, r_{1} / r_{0}$, and $F(h)$. From $(5.29)$ and (5.11) the claim follows.

Proposition 5.3. Under the same hypotheses of Proposition 5.2 we have

$$
\frac{\int_{D}|\nabla u|^{2}}{\int_{\Omega}|\nabla u|^{2}} \leq C r_{1}^{-n}|D|
$$

where $C$ depends only on $r_{1} / r_{0}, M_{0}, M_{1}, c_{0}$ and $L$.

Proof. Trivially we have

$$
\int_{D}|\nabla u|^{2} \leq|D|\|\nabla u\|_{L^{\infty}(D)}^{2} \leq|D|\|\nabla u\|_{L^{\infty}\left(\Omega \backslash B_{r_{1}}(P)\right)}^{2} .
$$

Since $\sigma_{0} \nabla u \cdot \nu=0$ on $\partial \Omega \backslash B_{r_{1} / 2}(P)$, from standard estimates for elliptic equations, [16], and from the Poincaré inequality, we have that, letting $\tau=\frac{1}{|\Omega|} \int_{\Omega} u$, there hold

$$
\begin{aligned}
\|\nabla u\|_{L^{\infty}\left(\Omega \backslash B_{r_{1}}(P)\right)}^{2} & \leq \frac{C_{1}}{r_{1}^{2}}\|u-\tau\|_{L^{\infty}\left(\Omega \backslash B_{\frac{3}{4} r_{1}}(P)\right)}^{2} \leq \frac{C_{1} C_{2}}{r_{1}^{n+2}}\|u-\tau\|_{L^{2}\left(\Omega \backslash B_{r_{1 / 2}}(P)\right)}^{2} \\
& \leq \frac{C_{1} C_{2}}{r_{1}^{n+2}}\|u-\tau\|_{L^{2}(\Omega)}^{2} \leq \frac{C_{1} C_{2} C_{3} r_{0}^{2}}{r_{1}^{n+2}}\|\nabla u\|_{L^{2}(\Omega)}^{2},
\end{aligned}
$$

where $C_{1}$ depends only on $r_{1} / r_{0}, M_{0}, M_{1}, c_{0}$ and $L ; C_{2}$ depends on $r_{1} / r_{0}, M_{0}, M_{1}$ and $c_{0}$; and $C_{3}$ depends on $M_{0}$ and $M_{1}$. From (5.30) and (5.31) we get

$$
\frac{\int_{D}|\nabla u|^{2}}{\int_{\Omega}|\nabla u|^{2}} \leq C_{4} r_{1}^{-n}|D|
$$

where $C_{4}$ depends only on $r_{1} / r_{0}, M_{0}, M_{1}, c_{0}$ and $L$.

We are now ready to prove our main results.

Proof of Theorem 2.3. By standard elliptic estimates, we have

$$
\sup _{D}\left|\nabla u_{0}\right| \leq C \sup _{\Omega_{d_{0} / 2}}\left|u_{0}\right| \leq C\left\|u_{0}\right\|_{L^{2}(\Omega)},
$$


From the trivial estimate

$$
\int_{\Omega}\left|\nabla u_{0}\right|^{2} \leq c_{0}^{-1} \int_{\Omega} \nabla u_{0} \cdot \nabla u_{0}=c_{0}^{-1} W_{0},
$$

and from the Poincaré inequality, we have

$$
\sup _{D}\left|\nabla u_{0}\right| \leq C W_{0}^{1 / 2}
$$

where $C$ depends on $c_{0}, L, d_{0} / r_{0}$, and $M_{0}$. Hence from (5.32) we get for $|D|$ the lower bound

$$
\int_{D}|\nabla u|^{2} \leq|D|\|\nabla u\|_{L^{\infty}(D)}^{2} \leq C|D| W_{0} .
$$

By Proposition 5.1 and (5.33) we obtain

$$
\tilde{C}_{1} \frac{\int_{D}\left|\nabla u_{0}\right|^{2}}{W_{0}} \leq \frac{|D|}{|\Omega|} \leq \tilde{C}_{2}\left(\frac{\int_{D}\left|\nabla u_{0}\right|^{2}}{W_{0}}\right)^{1 / p}
$$

where $\tilde{C}_{1}$ depends only on $d_{0} / r_{0}, M_{0}, M_{1}, c_{0}$ and $L$ and $\tilde{C}_{2}$ depends only on $d_{0} / r_{0}$, $M_{0}, M_{1}, c_{0}, L$ and $F(h)$. Finally, applying Proposition 3.3 if $\gamma_{0}, \gamma_{1}$ are constant and satisfy $(\mathbf{H 3 i})$ or applying Proposition 3.4 if $\gamma_{0}$ and $\gamma_{1}$ satisfy (H3ii), we get

$$
C_{1}\left|\frac{\delta W}{W_{0}}\right| \leq \frac{|D|}{|\Omega|} \leq C_{2}\left|\frac{\delta W}{W_{0}}\right|^{1 / p},
$$

where $C_{1}$ depends only on the a priori constants $c_{0}, \mu_{0}, M_{0}, M_{1}, d_{0} / r_{0}, L$, and the number $p>1$ and $C_{2}$ depends on the same parameters and $F(h)$.

Proof of Theorem 2.4. By Propositions 5.2 and 5.3 applied to the background potential $u_{0}$ that solves (2.3) (in the constant case up to a rescaling by a constant) we get

$$
C_{1}^{\prime} \frac{\int_{D}\left|\nabla u_{0}\right|^{2}}{W_{0}} \leq \frac{|D|}{|\Omega|} \leq C_{2}^{\prime}\left(\frac{\int_{D}\left|\nabla u_{0}\right|^{2}}{W_{0}}\right)^{1 / p}
$$

where $C_{1}^{\prime}$ depends only on $r_{1} / r_{0}, M_{0}, M_{1}, c_{0}$ and $L$ and $C_{2}^{\prime}$ depends on $r_{1} / r_{0}, M_{0}$, $M_{1}, c_{0}, L$, and $F(h)$. Finally applying Proposition 3.3 if $\gamma_{0}, \gamma_{1}$ are constant and satisfy (H3i) or applying Proposition 3.4 if $\gamma_{0}$ and $\gamma_{1}$ satisfy (H3ii) we get

$$
C_{1}\left|\frac{\delta W}{W_{0}}\right| \leq \frac{|D|}{|\Omega|} \leq C_{2}\left|\frac{\delta W}{W_{0}}\right|^{1 / p},
$$

where $C_{1}$ depends only on the a priori constants $c_{0}, \mu_{0}, M_{0}, M_{1}, r_{1} / r_{0}, L$, and the number $p>1$ only and $C_{2}$ depends on the same parameters and $F(h)$. 


\section{Appendix}

Proof of Theorem 4.3. The doubling inequality proved in [20] and [4] can be extended with straightforward arguments to the case of complex valued solutions of

$$
\operatorname{div}(A(x) \nabla u(x))=0 \quad \text { in } B_{r_{0}}\left(x_{0}\right) .
$$

We give an idea of the modifications that need to be done to the proof. We assume that

$$
A(0)=\mathrm{Id}
$$

and define, for $0<r<R_{0}$,

$$
H(r)=\int_{\partial B_{r}} \frac{A(x) x \cdot x}{|x|^{2}}|v(x)|^{2}, \quad I(r)=\int_{B_{r}} A(x) \nabla v \cdot \overline{\nabla v}, \quad N(r)=\frac{r I(r)}{H(r)}
$$

If, instead of Rellich's identity used in [20], we use the relation

$$
\begin{aligned}
& 2 \Re[(\beta \cdot \nabla \bar{v}) \operatorname{div}(A \nabla v)]=\operatorname{div}[2 \Re((\beta \cdot \nabla \bar{v}) A \nabla v)-\beta(A \nabla v \cdot \nabla \bar{v})] \\
& +(\operatorname{div} \beta) A \nabla v \cdot \nabla \bar{v}-2 \Re\left[\partial_{l} \beta_{j} a_{l k} \partial_{k} v \partial_{j} \bar{v}\right]+\beta_{j}\left(\partial_{j} a_{l k}\right) \partial_{k} v \partial_{l} \bar{v}
\end{aligned}
$$

with $\beta$ sufficiently smooth vector field on $\mathbb{R}^{n}$, we get that there exist constants $C_{1}>1, C_{2}$, and $c$, with $C_{1}>1, C_{2}$, depending only on $\lambda_{0}$ and $\lambda_{1}$ and $c$ an absolute constant, such that

$$
\begin{aligned}
& \int_{B_{r}}|v|^{2} \leq \lambda_{0}^{2} r \int_{\partial B_{r}}|v|^{2}, \quad \text { for } r \leq \frac{R_{0}}{C_{1}}, \\
& \left|H^{\prime}(r)-\frac{n-1}{r} H(r)-2 I(r)\right| \leq \frac{c \lambda_{1}}{R_{0}} H(r), \\
& N(r) e^{C_{2} r / R_{0}} \quad \text { increasing in }\left(0, R_{0}\right] .
\end{aligned}
$$

From (6.4) we have

$$
\begin{aligned}
& \frac{d}{d r}\left(\log \frac{H(r)}{r^{n-1}}\right) \leq \frac{c \lambda_{1}}{R_{0}}+\frac{2 N(r)}{r} \\
& \frac{2 N(r)}{r} \leq \frac{d}{d r}\left(\log \frac{H(r)}{r^{n-1}}\right)+\frac{c \lambda_{1}}{R_{0}}
\end{aligned}
$$

Let $R_{1}:=R_{0} / C_{1}$ and $\rho, R \in\left(0, R_{1}\right]$ be such that $3 \rho \leq R$. Integrating both sides of (6.6) in the interval $[\rho, 3 \rho]$ we get, using (6.5),

$$
\begin{aligned}
\log \frac{H(3 \rho)}{3^{n-1} H(\rho)} & \leq \frac{2 c \lambda_{1} \rho}{R_{0}}+\int_{\rho}^{3 \rho} \frac{2 N(r)}{r} \leq \frac{2 c \lambda_{1} \rho}{R_{0}}+\int_{\rho}^{3 \rho} \frac{2 N(r)}{r} e^{C_{2} r / R_{0}} \\
& \leq \frac{2 c \lambda_{1} \rho}{R_{0}}+2 N(3 \rho) e^{3 C_{2} \rho / R_{0}} \log 3 \leq \frac{2 c \lambda_{1} R}{3 R_{0}}+2 N(R) e^{3 C_{2} R / R_{0}} \log 3 .
\end{aligned}
$$


Hence, for $\rho \in(0, R / 3]$, and $R \in\left(0, R_{1}\right]$, one has

$$
\frac{1}{R} \log \frac{H(3 \rho)}{3^{n-1} H(\rho)} \leq \frac{2 c \lambda_{1}}{3 R_{0}}+2 e^{C_{2} R / R_{0}} \frac{N(R)}{R} \log 3 .
$$

From (6.8) and (6.7) we get, for $\rho \in\left(0, R_{1} / 3\right]$ and $R \in\left(0, R_{1}\right]$,

$$
\frac{1}{R} \log \frac{H(3 \rho)}{3^{n-1} H(\rho)} \leq \frac{C_{3}}{R_{0}}+e^{C_{2}}(\log 3) \frac{d}{d R}\left(\log \frac{H(R)}{R^{n-1}}\right),
$$

where $C_{3}=e^{C_{2} \log 3}+2 c \lambda_{1} / 3$. This last inequality implies, in particular, that for any $\rho \in\left(0, R_{1} / 9\right]$ and $R \in\left(R_{1} / 2,3 R_{1} / 4\right]$ one has (integrating both sides of $(6.9)$ over $\left.\left[R_{1} / 3, R\right]\right)$

$$
\begin{aligned}
\log 6 \log \frac{H(3 \rho)}{3^{n-1} H(\rho)} & \leq \log \frac{R}{R_{1} / 3} \int_{R_{1} / 3}^{R} \frac{1}{t} \log \frac{H(3 \rho)}{3^{n-1} H(\rho)} d t \\
& \leq C_{3} \lambda_{1} \frac{R-R}{R_{0}}+e^{C_{2}}(\log 3) \log \frac{H(R)}{\left(3 R / R_{1}\right)^{n-1} H\left(R_{1} / 3\right)} \\
& \leq C_{3} \lambda_{1}+e^{C_{2}}(\log 3) \log \frac{H(R)}{(3 / 2)^{n-1} H\left(R_{1} / 3\right)} .
\end{aligned}
$$

Hence, for $\rho \in\left(0, R_{1} / 9\right]$ and $R \in\left(R_{1} / 2,3 R_{1} / 4\right]$, by the elementary properties of the logarithm, we have,

$$
H(3 \rho) \leq C_{4}\left(\frac{H(R)}{H\left(R_{1} / 3\right)}\right)^{C_{5}} H(\rho),
$$

where $C_{4}$ and $C_{5}$ depend only on $\lambda_{0}$ and $\lambda_{1}$. Integrating both sides of (6.10), we derive, for every $\rho \in\left(0, R_{1} / 9\right]$ and $R \in\left(R_{1} / 2,3 R_{1} / 4\right]$,

$$
\int_{0}^{\rho} H(3 s) d s \leq C_{4}\left(\frac{H(R)}{H\left(R_{1} / 3\right)}\right)^{C_{5}} \int_{0}^{\rho} H(s) d s .
$$

From (6.2) we get

$$
\int_{0}^{\rho} H(s) d s \leq \lambda_{0}^{-1} \int_{B_{\rho}}|v|^{2} \text { and } \int_{0}^{\rho} H(3 s) d s \geq \frac{\lambda_{0}}{3} \int_{B_{3 \rho}}|v|^{2} .
$$

From the last two inequalities and from (6.10) one has

$$
\int_{B_{3 \rho}}|v|^{2} \leq 3 \lambda_{0}^{-2} C_{4}\left(\frac{H(R)}{H\left(R_{1} / 3\right)}\right)^{C_{5}} \int_{B_{\rho}}|v|^{2},
$$

for $\rho \in\left(0, R_{1} / 9\right]$ and $R \in\left(R_{1} / 2,3 R_{1} / 4\right]$.

Now, (6.11) holds also if instead of $v$ we insert $v-\tau_{\rho}$ where $\tau_{\rho}=\frac{1}{\left|B_{\rho}\right|} \int_{B_{\rho}} v$.

Denoting by $\tilde{H}(r)$ the function upon substituting $v-\tau_{\rho}$ for $v$ in (6.2), we have, recalling local boundness of solutions to elliptic equations, [16],

$$
\tilde{H}(R) \leq \lambda_{0}^{-2} \int_{\partial B_{R}}\left|v-\tau_{\rho}\right|^{2} \leq 4 \lambda_{0}^{-2} R^{n-1}\|v\|_{L^{\infty}\left(B_{R}\right)} \leq \frac{C}{R_{1}} \int_{B_{R_{1}}}|v|^{2},
$$


for every $R \in\left(R_{1} / 2,3 R_{1} / 4\right]$ where $C$ depends only on $\lambda_{0}$. On the other hand, applying (6.3), we obtain

$$
\tilde{H}\left(R_{1} / 3\right) \geq \lambda_{0} \int_{\partial B_{R_{1} / 3}}\left|v-\tau_{\rho}\right|^{2} \geq \int_{B_{R_{1} / 3}}\left|v-\tau_{\rho}\right|^{2} \geq \frac{R_{1}}{C} \int_{B_{R_{1} / 6}}|\nabla v|^{2},
$$

where $C \geq 1$ depends only on $\lambda_{0}$. From (6.12), (6.13), and (6.11) we get

$$
\int_{B_{3 \rho}}\left|v-\tau_{\rho}\right|^{2} \leq C_{6}\left(\frac{\int_{B_{R_{1}}}|v|^{2}}{R_{1}^{2} \int_{B_{R_{1} / 6}}|v|^{2}}\right)^{C_{5}} \int_{B_{\rho}}\left|v-\tau_{\rho}\right|^{2}
$$

where $C_{6} \geq 1$ depends on $\lambda_{0}, \lambda_{1}$. Using the Poincaré inequality and the Caccioppoli inequality to bound the right-hand side of (6.14) from above and the left-hand side of (6.14) from below, we obtain, for any $\rho \in\left(0, R_{1} / 3\right]$,

$$
\int_{B_{2 \rho}}|\nabla v|^{2} \leq C_{7}\left(\frac{\int_{B_{R_{1}}}|v|^{2}}{R_{1}^{2} \int_{B_{R_{1} / 6}}|\nabla v|^{2}}\right)^{C_{5}} \int_{B_{\rho}}|\nabla v|^{2},
$$

where $C_{6} \geq 1$ depends on $\lambda_{0}$ and $\lambda_{1}$.

Iterating (6.15), by simple calculations we get

$$
\int_{B_{\alpha \rho}}|\nabla v|^{2} \leq C_{8} N_{v}^{\prime} \alpha^{\log N_{v} / \log 2} \int_{B_{\rho}}|\nabla v|^{2},
$$

for any $\alpha \geq 1$ and $\rho$ such that $3 \alpha \rho \leq R_{1}$. Here we have set

$$
N_{v}^{\prime}=\left(\frac{\int_{B_{R_{1}}}|v|^{2}}{R_{1}^{2} \int_{B_{R_{1} / 6}}|\nabla v|^{2}}\right)^{C_{5}}
$$

and $C_{8}$ depends on $\lambda_{0}$ and $\lambda_{1}$ only. Now we remove condition (6.1). To this end, let $A(x)$ be a symmetric matrix satisfying (4.1) and (4.2) and let $v \in H^{1}\left(B_{R_{0}}\right)$ a weak solution of (4.4). Let us introduce the change of variables $y=J x$ where $J=\sqrt{A^{-1}(0)}$ and consider, for any $r>0$, the ellipsoids

$$
E_{r}:=\left\{x \in \mathbb{R}^{n}: A^{-1}(0) x \cdot x<r^{2}\right\}=J^{-1}\left(B_{r}\right) .
$$

Setting $w(y)=v\left(J^{-1} y\right)$ and $\tilde{A}(y)=J A\left(J^{-1} y\right) J$ one has

$$
\begin{aligned}
& \operatorname{div}\left(\tilde{A}(y) \nabla_{y} w(y)\right)=0 \quad \text { in } B_{R_{0} \sqrt{\lambda_{0}}}, \\
& \lambda_{0}^{2}|\xi|^{2} \leq \tilde{A}(y) \xi \cdot \xi \leq \lambda_{0}^{-2}|\xi|^{2}, \quad \forall y \in \mathbb{R}^{n}, \forall \xi \in \mathbb{R}^{n}, \\
& \left|\tilde{A}\left(y_{1}\right)-\tilde{A}\left(y_{2}\right)\right| \leq \frac{\lambda_{0}^{-3 / 2} \lambda_{1}}{R_{0}}\left|y_{1}-y_{2}\right|, \quad \forall y_{1}, y_{2} \in \mathbb{R}^{n} \text { and } \tilde{A}(0)=\mathrm{Id} .
\end{aligned}
$$

Furthermore, since

$$
B_{\sqrt{\lambda_{0}} r} \subset E_{r} \subset B_{r / \sqrt{\lambda_{0}}}, \quad \forall r>0,
$$

by simple changes of variables we have

$$
\lambda_{0}^{n / 2+1} \int_{B_{\sqrt{\lambda_{0}} r}}|\nabla v|^{2} d x \leq \int_{B_{r}}|\nabla w|^{2} d y \leq \lambda_{0}^{-(n / 2+1)} \int_{B_{r / \sqrt{\lambda_{0}}}}|\nabla v|^{2} d x .
$$


for all $r>0$. We apply (6.16) to $w$ and we obtain

$$
\int_{B_{\alpha \rho}}|\nabla w|^{2} d y \leq C_{8}^{\prime} N_{w}^{\prime \prime} \alpha^{\log N_{w}^{\prime \prime} / \log 2} \int_{B_{\rho}}|\nabla w|^{2} d y
$$

for any $\alpha \geq 1$ and $\rho$ such that $3 \alpha \rho \leq R_{1} \sqrt{\lambda_{0}}:=R_{2}$, where

$$
N_{w}^{\prime \prime}=\left(\frac{\int_{B_{R_{2}}}|w|^{2} d y}{R_{2}^{2} \int_{B_{R_{2} / 6}}|\nabla w|^{2}}\right)^{C_{5}^{\prime}}
$$

and $C_{5}^{\prime}$ and $C_{8}^{\prime}$ depend on $\lambda_{0}$ and $\lambda_{1}$ only. From (6.17) and (6.18) we derive easily

$$
\begin{aligned}
\int_{B_{\alpha \rho}}|\nabla v|^{2} d x & \leq \lambda_{0}^{-(n / 2+1)} \int_{B_{\alpha \rho / \sqrt{\lambda_{0}}}}|\nabla w|^{2} d y \\
& \leq C_{8}^{\prime} N_{w}^{\prime \prime}\left(\lambda_{0}^{-1} \alpha\right)^{\log N_{w}^{\prime \prime} / \log 2} \lambda_{0}^{-(n / 2+1)} \int_{B_{\rho \sqrt{\lambda_{0}}}}|\nabla w|^{2} d y \\
& \leq C_{8}^{\prime} \lambda_{0}^{-(n+2)} N_{w}^{\prime \prime}\left(\lambda_{0}^{-1} \alpha\right)^{\log N_{w}^{\prime \prime} / \log 2} \int_{B_{\rho}}|\nabla v|^{2} d x
\end{aligned}
$$

for any $\alpha \geq 1$ and $\rho$ such that $3 \alpha \rho \leq R_{2}$. From (6.19) and using (6.17) to estimate $N_{w}^{\prime \prime}$ in terms of $v$ we get

$$
\int_{B_{2 \rho}}|\nabla v|^{2} d x \leq C_{10}\left(\frac{\int_{B_{R_{1}}}|v|^{2}}{R_{1}^{2} \int_{B_{R_{1} \lambda_{0} / 6}}|\nabla v|^{2}}\right)^{C_{9}} \int_{B_{\rho}}|\nabla v|^{2} d x
$$

for any $\rho \leq R_{1} \lambda_{0} / 6$ and where $C_{9}$ and $C_{10}$ depend on $\lambda_{0}$ and $\lambda_{1}$ only.

Applying (6.20) to $v-\frac{1}{\left|B_{R_{1}}\right|} \int_{B_{R_{1}}} v$ and using the Poincaré inequality we have

$$
\int_{B_{2 \rho}}|\nabla v|^{2} d x \leq C_{11}\left(\frac{\int_{B_{R_{1}}}|\nabla v|^{2}}{R_{1}^{2} \int_{B_{R_{1} \lambda_{0} / 6}}|\nabla v|^{2}}\right)^{C_{9}} \int_{B_{\rho}}|\nabla v|^{2} d x
$$

for $\rho \leq R_{1} \lambda_{0} / 6$. Finally we want to prove (4.5). Let $\rho \in\left[R_{1} \lambda_{0} / 6, R_{0} / 2\right]$. We trivially have

$$
\begin{aligned}
\int_{B_{2 \rho}}|\nabla v|^{2} d x & \leq \int_{B_{R_{0}}}|\nabla v|^{2} d x=\left(\frac{\int_{B_{R_{0}}}|\nabla v|^{2} d x}{\int_{B_{R_{1} \lambda_{0} / 6}}|\nabla v|^{2} d x}\right) \int_{B_{R_{1} \lambda_{0} / 6}}|\nabla v|^{2} d x \\
& \leq\left.\left(\frac{\int_{B_{R_{0}}}|\nabla v|^{2} d x}{\int_{B_{R_{1} \lambda_{0} / 6}}|\nabla v|^{2} d x}\right) \int_{B_{\rho}} \nabla v\right|^{2} d x
\end{aligned}
$$

From last inequality and from (6.21) we immediately get, for $\rho \in\left[0, R_{0} / 2\right]$,

$$
\int_{B_{2 \rho}}|\nabla v|^{2} d x \leq C_{11}\left(\frac{\int_{B_{R_{0}}}|\nabla v|^{2} d x}{\int_{B_{R_{1} \lambda_{0} / 6}}|\nabla v|^{2} d x}\right) \int_{B_{\rho}}|\nabla v|^{2} d x .
$$


Now, we apply the three spheres inequality

$$
\int_{B_{R_{0} / 2}}|\nabla v|^{2} d x \leq C_{12}\left(\int_{B_{R_{0}}}|\nabla v|^{2} d x\right)^{\theta}\left(\int_{B_{R_{1} \lambda_{0} / 6}}|\nabla v|^{2} d x\right)^{1-\theta}
$$

where $C_{12}$ and $\theta \in(0,1)$ depend only on $\lambda_{0}$ and $\lambda_{1}$. From (6.23) we have trivially

$$
\frac{\int_{B_{R_{0}}}|\nabla v|^{2} d x}{\int_{B_{R_{1} \lambda_{0} / 6}}|\nabla v|^{2} d x} \leq C_{12}\left(\frac{\int_{B_{R_{0}}}|\nabla v|^{2} d x}{\int_{B_{R_{0} / 2}}|\nabla v|^{2} d x}\right)^{1 /(1-\theta)} .
$$

From this last inequality and (6.22) we finally get (4.5).

Acknowledgements. We thank Paolo Bisegna for having proposed the problem to one of the authors and pointing out the importance of this problem in applications. We want also to thank Micol Amar, Daniele Andreucci and Roberto Gianni for stimulating discussions on the problem.

\section{References}

[1] Adolfsson, V. and Escauriaza, L.: $C^{1, \alpha}$ domains and unique continuation at the boundary. Comm. Pure Appl. Math. 50 (1997), no. 10, 935-969.

[2] Alessandrini, G., Beretta, E., Rosset, E. and Vessella, S.: Optimal stability for inverse elliptic boundary value problems with unknown boundaries. Ann. Scuola Norm. Sup. Pisa Cl. Sci. (4) 29 (2000), no. 4, 755-806.

[3] Alessandrini, G., Morassi, A. and Rosset, E.: Size estimates. In Inverse Problems: Theory and Applications (Cortona/Pisa), 1-34. Contemp. Math. 333, Amer. Math. Soc., Providence, RI, 2003.

[4] Alessandrini, G., Rondi, L., Rosset, E. and Vessella, S.: The stability for the Cauchy problem for elliptic equations. Inverse Problems 25 (2009), no. 12, 1-47.

[5] Alessandrini, G. And Rosset, E.: The inverse conductivity problem with one measurement: bounds on the size of the unknown object. SIAM J. Appl. Math. 58 (1998), no. 4, 1060-1071.

[6] Alessandrini, G., Rosset, E. and Seo, J. K.: Optimal size estimates for the inverse conductivity problem with one measurement. Proc. Amer. Math. Soc. 128 (2000), no. 1, 53-64.

[7] Amar, M., Andreucci, D., Bisegna, P. and Gianni, R.: Homogenization for electrical conduction in biological tissues in the radio frequency range. Comptes Rendues Mecanique 331 (2003), 503-508.

[8] Amar, M., Andreucci, D., Bisegna, P. And Gianni, R.: Exponential asymptotic stability for an elliptic equation with memory arising in electrical conduction in biological tissues. European J. Appl. Math. 20 (2009), no. 5, 431-459.

[9] Borcea, L.: Electrical impedance tomography. Inverse Problems 18 (2002), no. 6, R99-R136.

[10] Capdeboscq, Y. and Vogelius, M.: Optimal asymptotic estimates for the volume of internal inhomogeneities in terms of multiple boundary measurements. M2AN Math. Model. Numer. Anal. 37 (2003), no. 2, 227-240. 
[11] Cheney, M., Isaacson, D. and Newell, J. C.: Electrical impedence tomography. SIAM Rev. 41 (1999), no. 1, 85-101.

[12] Cherkaev, A. V. and Gibiansky, L. V.: Variational principles for complex conductivity, viscoelasticiy, and similar problems in media with complex moduli. J. Math. Phys. 35 (1994), no. 1, 127-145.

[13] Coifman, R. and Fefferman, C.: Weighted norm inequalities for maximal functions and singular integrals. Studia Math. 51 (1974), 241-250.

[14] García-Cuerva, J. and Rubio de Francia, J. L.: Weighted norm inequalities and related topics. North-Holland Mathematics Studies 116, North Holland, Amsterdam, 1985.

[15] Garofalo, N. And Lin, F. H.: Monotonicity properties of variational integrals, $A_{p}$ weights and unique continuation. Indiana Univ. Math. J. 35 (1986), no. 2, 245-268.

[16] Gilbarg, D. and Trudinger, N. S.: Elliptic partial differential equations of second order. Grundleheren der Mathematischen Wissenshaften 224, Springer-Verlag, Berlin, 1983.

[17] Griffiths, H.: Tissue spectroscopy with electrical impedance tomography: computer simulations. IEEE Trans. Biomed. Eng. 42 (1995), 948-953.

[18] Kang, H., Kim, E. And Milton, G.: Sharp bounds on the volume fractions of two materials in a two-dimensional body from electrical boundary measurements: the translation method. Calc. Var. Partial Differential Equations 45 (2012), no. 3-4, 367-401.

[19] Kang, H., Seo, J. K. And Sheen, D.: The inverse conductivity problem with one measurement: stability and estimation of size. SIAM J. Math. Anal. 28 (1997), no. $6,1389-1405$.

[20] Kukavica, I.: Quantitative uniqueness for second order elliptic operators. Duke Math. J. 91 (1998), no. 2, 225-240.

[21] Vessella, S.: Quantitative continuation fron a measurable set of solutions of elliptic equations. Proc. Roy. Soc. Edinburgh Sect. A 130 (2000), no. 4, 909-923.

Received May 27, 2012.

Elena Beretta: Dipartimento di Matematica 'Francesco Brioschi', Politecnico di Milano, Piazza Leonardo da Vinci 32, 20133 Milano, Italy.

E-mail: elena.beretta@polimi.it

Elisa Francini: Dipartimento di Matematica e Informatica, Università di Firenze, viale Morgagni 67A, 50134 Firenze, Italy.

E-mail: elisa.francini@unifi.it

Sergio Vessella: Dipartimento di Matematica e Informatica, Università di Firenze, viale Morgagni 67A, 50134 Firenze, Italy.

E-mail: sergio.vessella@unifi.it

This work was partially supported by Miur grant PRIN 20089 PWTPS003. 